\title{
ON THE DYNAMICS OF ROOTS AND POLES FOR SOLUTIONS OF THE POLUBARINOVA-GALIN EQUATION
}

\author{
Björn Gustafsson and Yu-Lin Lin \\ KTH Royal Institute of Technology, Department of Mathematics \\ SE-100 44, Stockholm, Sweden; gbjorn@kth.se \\ KTH Royal Institute of Technology, Department of Mathematics \\ SE-100 44, Stockholm, Sweden; ylli@kth.se
}

\begin{abstract}
We study the dynamics of roots of $f^{\prime}(\zeta, t)$, where $f(\zeta, t)$ is a locally univalent polynomial solution of the Polubarinova-Galin equation for the evolution of the conformal map onto a Hele-Shaw blob subject to injection at one point. We give examples of the sometimes complicated motion of roots, but show also that the asymptotic behavior is simple. More generally we allow $f^{\prime}(\zeta, t)$ to be a rational function and give sharp estimates for the motion of poles and for the decay of the Taylor coefficients. We also prove that any global in time locally univalent solution actually has to be univalent.
\end{abstract}

\section{Introduction}

Polynomial, rational and logarithmic solutions of the Polubarinova-Galin equation for the conformal map onto a growing or shrinking Hele-Shaw blob of viscous fluid have been studied in many papers the last few decades, see for example [19], [3], [9], [14], [20], [1], [8], [2], [10], [18], [22], [12], [13], and also [7]. In [19] Bensimon and Shraiman set up the dynamical equations, in the polynomial case, for how the zeros of the derivative of the conformal map move in the complex plane, and they proposed studying the dynamics of the zeros. As far as we know nobody has yet undertaken this task to any substantial extent. The purpose of the present paper is to start investigations in such a direction.

The general setting is that $f(\cdot, t): \mathbf{D} \rightarrow \Omega(t) \subset \mathbf{C}$ is the time dependent normalized $\left(f(0, t)=0, f^{\prime}(0, t)>0\right)$ conformal map onto the fluid region $\Omega(t)$, which has a source of strength $q(t)>0$ at the origin. The evolution is then described by the Polubarinova-Galin equation

$$
\operatorname{Re}\left[\dot{f}(\zeta, t) \overline{\zeta f^{\prime}(\zeta, t)}\right]=q(t) \quad \text { for } \zeta \in \partial \mathbf{D} .
$$

doi:10.5186/aasfm.2013.3802

2010 Mathematics Subject Classification: Primary 30C15, 34M30, 37N10, 76D27.

Key words: Hele-Shaw flow, Laplacian growth, Polubarinova-Galin equation, Löwner-Kufarev equation, root dynamics, pole dynamics.

This work has been supported by visiting grants from the Royal Institute of Technology (Stockholm) and Academia Sinica at Taipei, and in addition by the European Science Foundation Research Networking Programme HCAA, the Mittag-Leffler Institute (Djursholm, Sweden) and the Göran Gustafsson Foundation.

The authors are grateful to Saleh Tanveer for illuminating discussions and to Govind Menon, who helped the second author start out on the subject. 
We focus mainly on the case that the derivative $g(\zeta, t)=f^{\prime}(\zeta, t)$ is a rational function, a property which is preserved in time under (1.1). Then $g(\zeta, t)$, and hence $f(\zeta, t)$, is completely determined by the zeros $\omega_{1}(t), \ldots, \omega_{m}(t)$ and poles $\zeta_{1}(t), \ldots, \zeta_{n}(t)$ of $g(\zeta, t)$, both located outside the unit disk, together with an overall scale factor $b(t)$ :

$$
g(\zeta, t)=b(t) \frac{\prod_{k=1}^{m}\left(\zeta-\omega_{k}(t)\right)}{\prod_{j=1}^{n}\left(\zeta-\zeta_{j}(t)\right)} .
$$

The motion of the poles $\zeta_{j}(t)$ has previously been studied, for example it is known [20], [7] that they always move away from the origin. In the present paper we obtain more precise estimates of their speed and locations. The zeros $\omega_{k}(t)$ show up a more complicated behavior than the poles, for example they may occasionally move towards the origin, and even reach the unit circle, with possible break down of the solution as the result. Zeros may also collide with each other or escape to infinity in finite time. However the asymptotic behavior is simple: if the solution does not break down, then as $t \rightarrow \infty$ the zeros move to infinity arranged asymptotically as the corners of a regular polygon of growing size.

Besides the zeros and poles we also study the asymptotics of the coefficients in the power series

$$
f(\zeta, t)=\sum_{j=1}^{\infty} a_{j}(t) \zeta^{j}
$$

in the case that $g(\zeta, t)$ is rational. The leading coefficient $a_{1}(t)>0$ increases to infinity as $t \rightarrow \infty$, in fact can be normalized for example so that $a_{1}(t)=e^{t}$ (which then fixes $q(t)$ ), while the others tend to zero very quickly:

$$
a_{j}(t)=O\left(\frac{1}{a_{1}(t)^{j}}\right) \quad(j \geq 2)
$$

as $t \rightarrow \infty$.

Much of the behavior of solutions of the Polubarinova-Galin equation (1.1) can be understood in terms of harmonic moments and quadrature identities. These provide enough conserved quantities to make the Hele-Shaw problem fully integrable, hence in principle algebraically solvable. In practice it is not that easy because of the degree of complexity of the integrals of motion when expressed for example in terms of the Taylor coefficients of $f(\zeta, t)$. However the conservation laws allow for natural concepts of weak solutions, and with such relaxed forms of solutions it is possible to let zeros of $g(\zeta, t)$ penetrate the unit circle and enter the unit disk. Non locally univalent solutions of this kind will be studied in a forthcoming paper [4], and part of the aim of the present paper is to set the stage for these future investigations.

The organization of the paper is as follows. Section 2 contains all necessary preliminaries, in particular we set up the notations to be used. In Section 3 we derive the dynamical equations for the zeros and poles of $g(\zeta, t)$ by first writing (1.1) as an equation in $g$ alone (see (3.3)) and then identifying the residues in this formula (Theorem 3.1). When everything is spelled out (Theorem 3.2 and 3.3) one gets a rather involved system of ordinary differential equations for $\omega_{k}(t), \zeta_{j}(t), b(t)$. From Theorem 3.3 we obtain precise estimates of the speeds of the poles (Corollary 3.1), and a kind of conservation law for the dynamics (Proposition 3.2).

The Polubarinova-Galin equation (1.1) is not sensitive for loss of univalence of the solution as long as it stays locally univalent. However, loss of univalence is always 
followed by loss also of local univalence and break down of the solution at a later, but finite, time (Theorem 4.1). In [4] we will show how it in such cases still can be continued as a kind of weak solution spreading on a branched Riemann surface.

In Section 5 we give several examples (Example 5.1, 5.2, 5.3) of the behavior of roots $\omega_{k}(t)$ in the polynomial case, for example collision of roots, plus two theorems asserting the previously mentioned asymptotic behavior. In the first (Theorem 5.1) it is assumed a priori that the solution is global in time, while in the second (Theorem 5.2) only sufficiently strong assumptions on the initial data are made. Section 6 , finally, contains precise estimates for the poles $\zeta_{j}(t)$ in the rational case (Theorem 6.1) as well as the asymptotic estimates (1.3) of the Taylor coefficients of $f(\zeta, t)$ (Theorem 6.2; see also Lemma 5.1). There are also some sharper estimates (e.g., Corollary 6.2) for the case that the sequence of harmonic moments contains gaps.

\section{Preparatory material}

2.1. List of notations. We first list some general notations which will be used in the paper.

- $\mathbf{D}=\{\zeta \in \mathbf{C}:|\zeta|<1\}, \mathbf{D}(a, r)=\{\zeta \in \mathbf{C}:|\zeta-a|<r\}$.

- $d m=d m(z)=d x d y=\frac{1}{2 \mathrm{i}} d \bar{z} d z(z=x+\mathrm{i} y)$, area measure in the $z$ plane.

- $\omega^{*}=\frac{1}{\omega}$, for $\omega \in \mathbf{C}$.

- $h^{*}(\zeta)=\overline{h(1 / \bar{\zeta})}=\sum_{j=1}^{m} \bar{b}_{j} \zeta^{-j}$, where $h(\zeta, t)=\sum_{j=1}^{m} b_{j} \zeta^{j}$. (There is a slight ambiguity in this notation: we have $\zeta^{*}=1 / \bar{\zeta}$ if $\zeta$ is considered as a point, whereas $f^{*}(\zeta)=1 / \zeta$ for the function $f(\zeta)=\zeta$.)

- $\dot{f}(\zeta, t)=\frac{\partial}{\partial t} f(\zeta, t), f^{\prime}(\zeta, t)=\frac{\partial}{\partial \zeta} f(\zeta, t)$.

- With $E \subset \mathbf{C}$ any set which contains the origin,

$$
\begin{aligned}
\mathcal{O}(E) & =\{f: f \text { is analytic in some neighborhood of } E\}, \\
\mathcal{O}_{\text {norm }}(E) & =\left\{f \in \mathcal{O}(E): f(0)=0, f^{\prime}(0)>0\right\}, \\
\mathcal{O}_{\text {locu }}(E) & =\left\{f \in \mathcal{O}_{\text {norm }}(E): f^{\prime} \neq 0 \text { on } E\right\}, \\
\mathcal{O}_{\text {univ }}(E) & =\left\{f \in \mathcal{O}_{\text {locu }}(E): f \text { is univalent (one-to-one) on } E\right\} .
\end{aligned}
$$

- If $a(t), b(t)$ are positive functions, $a(t) \sim b(t)$ will mean that there exist constants $0<c<C<\infty$ such that

$$
c \leq \frac{a(t)}{b(t)} \leq C
$$

for all $t$.

2.2. Basic equations. In the paper we shall study certain aspects of the Polubarinova-Galin equation, which describes the evolution of a simply connected Hele-Shaw fluid blob in the complex plane driven by injection or suction at one point, chosen to be the origin. A smooth map $t \mapsto f(\cdot, t) \in \mathcal{O}_{\text {univ }}(\overline{\mathbf{D}})$ will be called a (univalent) solution of the Polubarinova-Galin equation if it satisfies

$$
\operatorname{Re}\left[\dot{f}(\zeta, t) \overline{\zeta f^{\prime}(\zeta, t)}\right]=q(t) \quad \text { for } \zeta \in \partial \mathbf{D}
$$

in the pointwise sense. Here $q(t)$ is a real-valued continuous function, which is given in advance and which represents the strength of the source/sink at the origin. Typically 
$q= \pm 1$, which corresponds to injection (plus sign) or suction (minus sign) at a rate $2 \pi$. Since the transformation $t \mapsto-t$ changes $q$ to $-q$ in (2.1) it is enough to discuss one of the cases $q>0$ and $q<0$. In general we shall take $q>0$.

Equation (2.1) expresses that the image domains $\Omega(t)=f(\mathbf{D}, t)$ evolve in such a way that

$$
\frac{d}{d t} \int_{\Omega(t)} h d m=2 \pi q(t) h(0)
$$

for every function $h$ harmonic in a neighborhood of $\overline{\Omega(t)}$. This means that the speed of the boundary $\partial \Omega(t)$ in the normal direction equals $q(t)$ times the normal derivative of the Green's function of $\Omega(t)$ with a pole at $z=0$. The terminology Laplacian growth is also used for this kind of evolution. The equivalence between (2.1) and (2.2) follows from the general formula

$$
\frac{d}{d t} \int_{\Omega(t)} \varphi d m=\int_{\partial \mathbf{D}} \varphi(f(\zeta, t)) \operatorname{Re}\left[\dot{f}(\zeta, t) \overline{\zeta f^{\prime}(\zeta, t)}\right] d \theta \quad\left(\zeta=e^{\mathrm{i} \theta}\right)
$$

valid for any smooth evolution $t \mapsto f(\cdot, t) \in \mathcal{O}_{\text {univ }}(\overline{\mathbf{D}})$ and for any smooth test function $\varphi$ in the complex plane. The derivation of (2.3) is straightforward (and omitted).

On choosing $h(z)=z^{k}, k=0,1,2, \ldots$, in $(2.2)$ it follows that the harmonic moments

$$
M_{k}(t)=\frac{1}{\pi} \int_{\Omega(t)} z^{k} d m=\frac{1}{2 \pi \mathrm{i}} \int_{\partial \mathbf{D}} f(\zeta, t)^{k} f^{*}(\zeta, t) f^{\prime}(\zeta, t) d \zeta
$$

are conserved quantities, except for the first one, which is related to $q$ by $\frac{d}{d t} M_{0}(t)=$ $2 q(t)$. Thus

$$
M_{0}(t)=M_{0}(0)+2 Q(t),
$$

where $Q(t)$ is the accumulated source up to time $t>0$ :

$$
Q(t)=\int_{0}^{t} q(s) d s
$$

The preservation of $M_{1}, M_{2}, \ldots$ characterizes Laplacian growth in the simply connected case.

One may consider the equation (2.1) on different levels of generality. It is natural to keep the normalization $f(0)=0, f^{\prime}(0)>0$, in fact the coupling to (2.2) depends on this, but (2.1) makes sense for any $f \in \mathcal{O}_{\text {norm }}(\overline{\mathbf{D}})$, at least as long one makes sure that $q(t)=0$ whenever a zero of $f^{\prime}$ appears on $\partial \mathbf{D}$. In a forthcoming paper [4] we shall deal with this general case, while in the present paper we shall only consider locally univalent functions, $f \in \mathcal{O}_{\text {locu }}(\overline{\mathbf{D}})$. The mathematical treatment of $(2.1)$ in this case is exactly the same as in the 'physical' case $f \in \mathcal{O}_{\text {univ }}(\overline{\mathbf{D}})$. We shall then speak of a locally univalent solution of the Polubarinova-Galin equation.

When $f \in \mathcal{O}_{\text {locu }}(\overline{\mathbf{D}})$, then $\dot{f} / \zeta f^{\prime} \in \mathcal{O}(\overline{\mathbf{D}})$ and equation (2.1) can be solved for $\dot{f}$ by dividing both sides with $\left|\zeta f^{\prime}\right|^{2}$. The result is an equation which we shall refer to as the Löwner-Kufarev equation, namely

$$
\dot{f}(\zeta, t)=\zeta f^{\prime}(\zeta, t) P(\zeta, t) \quad(\zeta \in \mathbf{D}),
$$


where $P(\zeta, t)$ is the analytic function in $\mathbf{D}$ whose real part has boundary value $q(t)\left|f^{\prime}(\zeta, t)\right|^{-2}$ and which is normalized by $\operatorname{Im} P(0, t)=0$. Explicitly $P(\zeta, t)$ is given by

$$
P(\zeta, t)=\frac{1}{2 \pi i} \int_{\partial \mathbf{D}} \frac{q(t)}{\left|f^{\prime}(z, t)\right|^{2}} \frac{z+\zeta}{z-\zeta} \frac{d z}{z} \quad(\zeta \in \mathbf{D})
$$

The right member in (2.7) is the Poisson-Schwarz integral

$$
\mathcal{P}_{\mu}(\zeta)=\int_{0}^{2 \pi} \frac{e^{i \theta}+\zeta}{e^{i \theta}-\zeta} d \mu(\theta) \quad(\zeta \in \mathbf{D})
$$

for the measure

$$
d \mu(\theta, t)=\frac{q(t)}{\left|f^{\prime}\left(e^{i \theta}, t\right)\right|^{2}} \frac{d \theta}{2 \pi} .
$$

If $q(t)>0$, then $\mu$ is positive and $\operatorname{Re} P>0$ in $\mathbf{D}$.

Remark 2.1. In the general literature on univalent functions, "Löwner-Kufarev equation" usually refers to any equation of the type (2.6) without any particular coupling between $f$ and $P$. The Hele-Shaw case is characterized by the feed-back relation (2.7) between $f$ and $P$.

Expanding $f$ in a power series,

$$
f(\zeta, t)=a_{1}(t) \zeta+a_{2}(t) \zeta^{2}+\ldots,
$$

it follows from (2.6) that

$$
\frac{\dot{a}_{1}(t)}{a_{1}(t)}=P(0, t)=\int d \mu(\cdot, t) .
$$

In particular, if $q(t)$ is chosen so that $\mu$ becomes a probability measure, namely

$$
\frac{1}{q(t)}=\frac{1}{2 \pi} \int_{0}^{2 \pi} \frac{d \theta}{\left|f^{\prime}\left(e^{\mathrm{i} \theta}, t\right)\right|^{2}},
$$

then $P(0, t)=1$ and $a_{1}(t)=a_{1}(0) e^{t}$.

When $f \in \mathcal{O}_{\text {locu }}(\overline{\mathbf{D}})$ then $P \in \mathcal{O}(\overline{\mathbf{D}})$, in fact the right member of (2.6) extends analytically as far as $f$ does (see [3]). We shall keep the notation $P=P(\zeta, t)$ also for the analytic extension of the Poisson integral beyond $\overline{\mathbf{D}}$.

As a general notation throughout the paper, we set

$$
g(\zeta, t)=f^{\prime}(\zeta, t)
$$

The function $g$ in fact turns out to be more fundamental than $f$ itself. Of course, $f$ can be recaptured from $g$ by

$$
f(z, t)=\int_{0}^{z} g(\zeta, t) d \zeta
$$

Most of the paper will deal with the case that $g$ is a rational function, or perhaps better to say, $g(\zeta) d \zeta$ is a rational differential, in other words an Abelian differential on the Riemann sphere. If $g$ has residues then $f$ will have logarithmic poles, besides ordinary poles. The terminology Abelian domain for the image domain $\Omega=f(\mathbf{D})$ has been used [21] for this case. Alternatively one may speak of $\Omega$ being a quadrature 
domain (see [6] for the terminology), which in the present case means that a finite quadrature identity of the kind

$$
\int_{\Omega} h(z) d x d y=\sum_{j=1}^{r} c_{j} \int_{\gamma_{j}} h(z) d z+\sum_{j=0}^{\ell} \sum_{k=1}^{n_{j}-1} a_{j k} h^{(k-1)}\left(z_{j}\right)
$$

holds for integrable analytic functions $h$ in $\Omega$. Here the points $z_{j} \in \Omega$ are fixed (i.e., independent of $h$ ), with specifically $z_{0}=0$, the $c_{j}, a_{j k}$ are fixed coefficients, and the $\gamma_{j}$ are arcs in $\Omega$ with end points among the $z_{j}$. This sort of structure is stable under Hele-Shaw flow because, as is seen from (2.2), what happens under the evolution is only that the right member is augmented by the term $2 \pi Q(t) h(0)$.

When $g=f^{\prime}$ is rational we shall write it on the form

$$
g(\zeta, t)=b(t) \frac{\prod_{k=1}^{m}\left(\zeta-\omega_{k}(t)\right)}{\prod_{j=1}^{n}\left(\zeta-\zeta_{j}(t)\right)}=b(t) \frac{\prod_{i=1}^{m}\left(\zeta-\omega_{i}(t)\right)}{\prod_{j=1}^{\ell}\left(\zeta-\zeta_{j}(t)\right)^{n_{j}}} .
$$

Here $m \geq n=\sum_{j=1}^{\ell} n_{j},\left|\zeta_{j}\right|>1$ and repetitions are allowed among the $\omega_{k}, \zeta_{j}$ to account for multiple zeros and poles. Then, with the argument of $b(t)$ chosen so that $g(0, t)>0, f \in \mathcal{O}_{\text {locu }}(\overline{\mathbf{D}})$ if and only if $\left|\omega_{k}\right|>1,\left|\zeta_{j}\right|>1$ for all $k$ and $j$. The assumption $m \geq n$ means that $g(\zeta) d \zeta$, as a differential, has at least a double pole at infinity, which the Hele-Shaw evolution in any case will force it to have because the source/sink at the origin creates a pole of $f$ at infinity.

The form (2.15) is stable in time, with the sole exception that when $m=n$ the pole of $f$ at infinity may disappear at one moment of time. Then the value of $m$ drops below $n$ at this moment. This can easily be explained in terms of the quadrature identity (2.14): under the Hele-Shaw evolution there will be one time dependent term, namely $\left(a_{01}+2 \pi Q(t)\right) h(0)$, and this may vanish for one value of $t$. If there are no terms $a_{0 k} h^{(k-1)}(0)$ with $k \geq 2$ this will cause $f$ to temporarily lose its pole at infinity. The phenomenon is illustrated in Example 3.2 below.

The rightmost member of (2.15) will be used when we need to be explicit about the orders of the poles. The convention then is that $\zeta_{1}, \ldots, \zeta_{\ell}$ are distinct and $n_{j} \geq 1$. Thus $n=\sum_{j=1}^{\ell} n_{j}$, and in the full sequence $\zeta_{1}, \ldots, \zeta_{n}$, the tail $\zeta_{\ell+1}, \ldots, \zeta_{n}$ will be repetitions of (some of) the $\zeta_{1}, \ldots, \zeta_{\ell}$ according to their orders. In equations (2.14) and (2.15), $\ell$ and the $n_{j}$ are the same.

For later use we here also exhibit $\log g$ and its derivatives:

$$
\begin{gathered}
\log g(\zeta, t)=\log b(t)+\sum_{k=1}^{m} \log \left(\zeta-\omega_{k}(t)\right)-\sum_{j=1}^{n} \log \left(\zeta-\zeta_{j}(t)\right), \\
\frac{\partial}{\partial t} \log g(\zeta, t)=\frac{\dot{b}(t)}{b(t)}-\sum_{k=1}^{m} \frac{\dot{\omega}_{k}(t)}{\zeta-\omega_{k}(t)}+\sum_{j=1}^{n} \frac{\dot{\zeta}_{j}(t)}{\zeta-\zeta_{j}(t)}, \\
\frac{\partial}{\partial \zeta} \log g(\zeta, t)=\sum_{k=1}^{m} \frac{1}{\zeta-\omega_{k}(t)}-\sum_{j=1}^{n} \frac{1}{\zeta-\zeta_{j}(t)} .
\end{gathered}
$$

We remark that $\log |g|$ can be viewed as the logarithmic potential of the charge distribution which puts positive unit charges at the zeros of $g$, negative unit charges 
at the poles (added up according to multiplicities). This is the charge distribution corresponding to the divisor $(g)$ of $g$, which is defined as the formal linear combination

$$
(g)=\sum_{k=1}^{m} 1 \cdot\left(\omega_{k}\right)-\sum_{j=1}^{n} 1 \cdot\left(\zeta_{j}\right)-(m-n) \cdot(\infty) .
$$

(This is the divisor of $g$ as a function, the divisor of $g d \zeta$ as a differential has two additional poles at infinity.) One main subject of the present paper is the study of the dynamics of $(g)$ under Hele-Shaw flow.

Note that $\log |g|$, and even its restriction to $\partial \mathbf{D}$, contains all information of $f$. In fact, given $u=\log |g|$ on $\partial \mathbf{D}$ we can extend it harmonically to $\mathbf{D}$, then form its harmonic conjugate $v$, normalized by $v(0)=0$, and finally define $f$ by

$$
f(\zeta)=\int_{0}^{\zeta} \exp (u(z)+\mathrm{i} v(z)) d z
$$

Note also that $\left.(\log |g|)\right|_{\partial \mathbf{D}}$ is a "free" function, i.e., is subject to no constraints besides regularity (real analyticity is needed to start a Hele-Shaw evolution in a full neighborhood of $t=0$, see e.g. [16]).

The structure (2.15) of $g$ means that $f$ is of the form

$$
f(\zeta, t)=\sum_{j=1}^{\ell} e_{j} \log \left(\zeta-\zeta_{j}(t)\right)+\sum_{j=1}^{\ell} \sum_{k=1}^{n_{j}-1} \frac{c_{j k}(t)}{\left(\zeta-\zeta_{j}(t)\right)^{k}}+\sum_{k=0}^{m-n+1} d_{k}(t) \zeta^{k} .
$$

The coefficients $e_{j}$, which are the residues of $g(\zeta) d \zeta$, will not depend on time (this is a consequence of (2.1)). If they are not all zero, then $f$ is single-valued only outside a suitable system of 'cuts' in $\mathbf{C} \backslash \overline{\mathbf{D}}$ connecting the logarithmic poles. Note that if $\sum_{j=1}^{\ell} e_{j} \neq 0$ then $f$ automatically has a logarithmic pole at infinity, hence one of the cuts has to reach infinity in this case. The relationship between (2.20) and (2.14) for $\Omega=\Omega(t)=f(\mathbf{D}, t)$ is that $z_{j}=f\left(\zeta_{j}^{*}(t), t\right), 1 \leq j \leq \ell, z_{0}=f\left(\infty^{*}, t\right)=f(0, t)=0$, and that the above mentioned 'cuts' can be taken to be the reflections in the unit circle of the $\operatorname{arcs} f^{-1}\left(\gamma_{j}\right)$. Moreover, $\sum_{j=1}^{\ell} n_{j}=n$ and $n_{0}=m-n+2$, hence $\sum_{j=0}^{\ell} n_{j}=m+2$. One may also note that $b=(m-n+1) d_{m-n+1}$. The two systems of coefficients, $\left\{c_{j}, a_{j k}\right\}$ and $\left\{e_{j}, c_{j k}, d_{k}\right\}$, are related by nonlinear equations (see e.g. [21]).

\section{Dynamics of zeros and poles in the rational case}

In this section we set up the dynamical equations for zeros and poles in the locally univalent rational case. We take $f$ and $g$ to be of the form (2.20), (2.15), so that the divisor of $g$ is given by (2.19). When $g$ is rational also $P$ is rational. When we refer to $P$ outside $\mathbf{D}$ we shall always mean this rational function, or more generally the analytic continuation of $\left.P\right|_{\mathbf{D}}$ (when it exists). First we write the dynamical equations in a general form.

Theorem 3.1. Under the above assumptions, the evolution of the divisor $(g)$ under Hele-Shaw flow governed by the Löwner-Kufarev equation (2.6) is given by

$$
\dot{\omega}_{k}(t)=-\operatorname{Res}_{\zeta=\omega_{k}}\left[\zeta P(\zeta, t) \frac{g^{\prime}(\zeta, t)}{g(\zeta, t)}\right]
$$




$$
\dot{\zeta}_{j}(t)=\operatorname{Res}_{\zeta=\zeta_{j}}\left[\zeta P(\zeta, t) \frac{g^{\prime}(\zeta, t)}{g(\zeta, t)}\right],
$$

valid whenever $\omega_{k}, \zeta_{j}$ is a simple zero/pole (respectively) of $g$.

If $\omega_{k}$ is a multiple zero, with say $\omega_{\ell}=\omega_{k}$ for $\ell$ in an index set $K \subset\{1,2, \ldots, m\}$ (containing $k$ ), then the individual $\dot{\omega}_{k}(t)$ need not exist, but we have instead

$$
\frac{d}{d t} \sum_{\ell \in K} \omega_{\ell}(t)=-\operatorname{Res}_{\zeta=\omega_{k}}\left[\zeta P(\zeta, t) \frac{g^{\prime}(\zeta, t)}{g(\zeta, t)}\right] .
$$

For multiple poles the $\dot{\zeta}_{j}$ do exist, and we have

$$
n_{j} \dot{\zeta}_{j}(t)=\operatorname{Res}_{\zeta=\zeta_{j}}\left[\zeta P(\zeta, t) \frac{g^{\prime}(\zeta, t)}{g(\zeta, t)}\right]
$$

for $1 \leq j \leq \ell$.

Proof. From (2.6), i.e., $\dot{f}=\zeta g P$, we get $\dot{g}=(\zeta g P)^{\prime}$ and hence

$$
\frac{\partial}{\partial t} \log g(\zeta, t)=\zeta P(\zeta, t) \frac{\partial}{\partial \zeta} \log g(\zeta, t)+\frac{\partial}{\partial \zeta}(\zeta P(\zeta, t))
$$

At first this equality holds in $\mathbf{D}$, but since both members are rational functions it becomes an identity between two rational functions.

From $(2.17)$ we see that the $\dot{\omega}_{k}$ and $\dot{\zeta}_{j}$ are the residues of $\frac{\partial}{\partial t} \log g$. Since the last term in (3.3), being a total derivative, has no residues we immediately get the equations for $\dot{\omega}_{k}$ and $\dot{\zeta}_{j}$. The statement concerning multiple roots and poles also follows easily.

Remark 3.1. It should be mentioned that multiple zeros never survive for any period of time, only collisions can occur. Multiple poles are however stable, they never split or collide.

In addition to depending on the zeros and poles, $g$ in (2.15) also depends on the factor $b$. The $m+n+1$ complex parameters $\omega_{1}, \ldots, \omega_{m}, \zeta_{1}, \ldots, \zeta_{n}, b$ are connected to the coefficient $a_{1}(t)=g(0, t)>0$ by

$$
(-1)^{m-n} b \frac{\prod_{k=1}^{m} \omega_{k}}{\prod_{j=1}^{n} \zeta_{j}}=a_{1}
$$

In particular, the imaginary part of the left member vanishes, which means that the mentioned parameters are subject to one real constraint. Taking the logarithmic time derivative of (3.4) and using (2.11) give an evolution equation for $b(t)$ :

$$
\frac{d}{d t} \log b(t)=\int d \mu(t)-\frac{d}{d t} \sum_{k=1}^{m} \log \omega_{k}(t)+\frac{d}{d t} \sum_{j=1}^{n} \log \zeta_{j}(t) .
$$

We now proceed to evaluate the Poisson integral $P(\zeta, t)$ in (3.1). This can be done by a simple residue calculus in (2.7), using that $|g(\zeta, t)|^{2}=g(\zeta, t) g^{*}(\zeta, t)$ is a rational function in $\zeta$. The calculation becomes even more transparent if everything is done at an algebraic level, by which it essentially reduces to an expansion in partial fractions. 
The rational function $q(t) / g(\zeta, t) g^{*}(\zeta, t)$ has poles at the zeros of $g$ and $g^{*}$, i.e., at $\omega_{1}, \ldots, \omega_{m}, \omega_{1}^{*}, \ldots, \omega_{m}^{*}$. At infinity it has the behavior (by $(2.15)$ )

$$
\lim _{\zeta \rightarrow \infty} \frac{q(t)}{g(\zeta, t) g^{*}(\zeta, t)}=A_{\infty}= \begin{cases}\frac{q \prod_{j=1}^{n} \bar{\zeta}_{j}}{|b|^{2} \prod_{j=1}^{m} \bar{\omega}_{j}}=\frac{q}{b a_{1}} & \text { if } m=n, \\ 0 & \text { if } m>n .\end{cases}
$$

Assuming for simplicity that the roots $\omega_{1}, \ldots, \omega_{m}$ are distinct it follows that

$$
\frac{q(t)}{g(\zeta, t) g^{*}(\zeta, t)}=A_{\infty}+\sum_{k=1}^{m} \frac{\bar{A}_{k}}{\bar{\omega}_{k}}+\sum_{k=1}^{m}\left[\frac{A_{k}}{\zeta-\omega_{k}}+\frac{\bar{A}_{k} \zeta}{1-\bar{\omega}_{k} \zeta}\right],
$$

where the coefficients $A_{k}=A_{k}\left(q, b, \omega_{1}, \ldots, \omega_{m}, \zeta_{1}, \ldots, \zeta_{n}\right)$ are given by

$$
A_{k}=\frac{q(t)}{g^{\prime}\left(\omega_{k}, t\right) g^{*}\left(\omega_{k}, t\right)}=\frac{q}{|b|^{2}} \cdot \frac{\prod_{j}\left(\omega_{k}-\zeta_{j}\right) \prod_{j} \overline{\left(\omega_{k}^{*}-\zeta_{j}\right)}}{\prod_{j \neq k}\left(\omega_{k}-\omega_{j}\right) \prod_{j} \overline{\left(\omega_{k}^{*}-\omega_{j}\right)}}
$$

for $1 \leq k \leq m$. Note that $A_{k} \neq 0$ (when $\left.f \in \mathcal{O}_{\text {locu }}(\overline{\mathbf{D}})\right)$.

Now, $P(\zeta, t)$ is by definition $(2.7)$ that holomorphic function in $\mathbf{D}$ whose real part has boundary values $q(t) / g(\zeta, t) g^{*}(\zeta, t)$ and whose imaginary part vanishes at the origin. The function (3.7) itself certainly has the right boundary behaviour on $\partial \mathbf{D}$, but it is not holomorphic in $\mathbf{D}$. On the other hand, the two types of polar parts occurring in (3.7) have the same real parts on the boundary:

$$
\operatorname{Re} \frac{A_{k}}{\zeta-\omega_{k}}=\operatorname{Re} \frac{\bar{A}_{k} \zeta}{1-\bar{\omega}_{k} \zeta} \quad \text { on } \partial \mathbf{D}
$$

Therefore, without changing the real part on the boundary we can make the function (3.7) holomorphic in $\mathbf{D}$ by a simple exchange of polar parts. In addition, one can add a purely imaginary constant to account for the normalization of $P$ at the origin. The result is that

$$
P(\zeta, t)=A_{0}+\sum_{j=1}^{m} \frac{2 A_{j}}{\zeta-\omega_{j}}
$$

for a suitable constant $A_{0}$. Since $P(0, t)=\int d \mu$ is real, the imaginary part of $A_{0}$ is given by

$$
\operatorname{Im} A_{0}=\operatorname{Im} \sum_{j=1}^{m} \frac{2 A_{j}}{\omega_{j}} .
$$

For the reflected kernel $P^{*}(\zeta, t)=\overline{P(1 / \bar{\zeta}, t)}$ we get

$$
P^{*}(\zeta, t)=\bar{A}_{0}+\sum_{j=1}^{m} \frac{2 \bar{A}_{j} \zeta}{1-\bar{\omega}_{j} \zeta} .
$$

On the other hand, the boundary condition on $\partial \mathbf{D}$ satisfied by $\operatorname{Re} P$ shows that

$$
P(\zeta, t)+P^{*}(\zeta, t)=\frac{2 q(t)}{g(\zeta, t) g^{*}(\zeta, t)}
$$


identically as rational functions. Therefore we find, on comparing (3.9), (3.11), (3.7) with (3.12) and using (3.10), that $A_{0}$ is given by

$$
A_{0}=A_{\infty}+\sum_{j=1}^{m} \frac{A_{j}}{\omega_{j}} .
$$

This also shows that

$$
A_{\infty}-\sum_{j=1}^{m} \frac{A_{j}}{\omega_{j}}=A_{0}-\sum_{j=1}^{m} \frac{2 A_{j}}{\omega_{j}}=P(0)=\int d \mu .
$$

In particular, if $m>n$, so that $A_{\infty}=0$, we have

$$
A_{0}=\sum_{j=1}^{m} \frac{A_{j}}{\omega_{j}}=-\int d \mu .
$$

Now we evaluate the residues in (3.1):

$$
\begin{aligned}
& \operatorname{Res}_{\zeta=\omega_{k}}\left[\zeta P(\zeta) \frac{g^{\prime}(\zeta)}{g(\zeta)}\right] \\
& =\operatorname{Res}_{\zeta=\omega_{k}}\left(A_{0} \zeta+\sum_{j=1}^{m} 2 A_{j}+\sum_{j=1}^{m} \frac{2 A_{j} \omega_{j}}{\zeta-\omega_{j}}\right)\left(\sum_{j=1}^{m} \frac{1}{\zeta-\omega_{j}}-\sum_{j=1}^{n} \frac{1}{\zeta-\zeta_{j}}\right) \\
& =A_{0} \omega_{k}+2 A_{k}+\sum_{j=1, j \neq k}^{m} \frac{2\left(A_{k}+A_{j}\right) \omega_{k}}{\omega_{k}-\omega_{j}}-\sum_{j=1}^{n} \frac{2 A_{k} \omega_{k}}{\omega_{k}-\zeta_{j}} .
\end{aligned}
$$

For the poles of $g$ the calculation of the residues is simpler because $P(\zeta)$ is regular there: we simply have

$$
\operatorname{Res}_{\zeta=\zeta_{j}}\left[\zeta P(\zeta) \frac{g^{\prime}(\zeta)}{g(\zeta)}\right]=-n_{j} \zeta_{j} P\left(\zeta_{j}\right),
$$

where $n_{j}$ is the order of the pole $\zeta_{j}$.

We summarize:

Theorem 3.2. In the case of simple zeros of $g$ we have the rational dynamics

$$
\begin{aligned}
-\frac{d}{d t} \log \omega_{k} & =A_{0}+\frac{2 A_{k}}{\omega_{k}}+\sum_{j=1, j \neq k}^{m} \frac{2\left(A_{k}+A_{j}\right)}{\omega_{k}-\omega_{j}}-\sum_{j=1}^{n} \frac{2 A_{k}}{\omega_{k}-\zeta_{j}} \\
-\frac{d}{d t} \log \zeta_{j} & =A_{0}+\sum_{k=1}^{m} \frac{2 A_{k}}{\zeta_{j}-\omega_{k}} \\
\frac{d \log b}{d t} & =(m-n+1) A_{0}
\end{aligned}
$$

where the $A_{j}$ are given by (3.6), (3.8), (3.13).

The last equation follows by letting $\zeta \rightarrow \infty$ in (3.3) and using (2.17), (2.18) and (3.9). The equation (3.16) was obtained, in the polynomial case $(n=0)$, already in [19]. 
It is useful to observe that the reflected Poisson integral $P^{*}(\zeta, t)=\overline{P(1 / \bar{\zeta}, t)}$ is nothing else than the corresponding Poisson integral for the exterior domain $\mathbf{P} \backslash \overline{\mathbf{D}}$ :

$$
P^{*}(\zeta, t)=\frac{1}{2 \pi i} \int_{\partial(\mathbf{P} \backslash \overline{\mathbf{D}})} \frac{q(t)}{|g(z, t)|^{2}} \frac{z+\zeta}{z-\zeta} \frac{d z}{z} \quad(\zeta \in \mathbf{P} \backslash \overline{\mathbf{D}}) .
$$

This follows from (2.7) and (3.12) by taking into account that the jump of the Poisson integral when $\zeta$ crosses the unit circle is exactly the right member in (3.12). The orientation of the unit circle in (3.19) is the opposite of that in (2.7). Note that $P^{*}>0$ in $\mathbf{P} \backslash \overline{\mathbf{D}}$ with $P^{*}(\infty)=P(0)=\int d \mu$, and also that the right member in (3.12) vanishes at the poles $\zeta_{j}$ of $g$, hence that

$$
P^{*}\left(\zeta_{j}, t\right)=-P\left(\zeta_{j}, t\right)
$$

Next we recast parts of Theorem 3.2 more directly in terms of the exterior Poisson integral:

Theorem 3.3. In the case of simple zeros of $g$ we have

$$
\begin{aligned}
\frac{d}{d t} \log \omega_{k} & =P^{*}\left(\omega_{k}, t\right)-\frac{2 A_{k}}{\omega_{k}}\left(1+\sum_{j=1}^{m} \frac{1}{1-\bar{\omega}_{j} \omega_{k}}-\sum_{j=1}^{n} \frac{1}{1-\bar{\zeta}_{j} \omega_{k}}\right), \\
\frac{d}{d t} \log \zeta_{j} & =P^{*}\left(\zeta_{j}, t\right) .
\end{aligned}
$$

For later use we recall (see (3.8)) that

$$
\frac{2 A_{k}}{\omega_{k}}=\frac{2 q}{\omega_{k} g^{\prime}\left(\omega_{k}, t\right) g^{*}\left(\omega_{k}, t\right)} .
$$

Proof. Set

$$
R(\zeta, t)=\frac{2 q(t)}{g(\zeta, t) g^{*}(\zeta, t)}
$$

so that $P+P^{*}=R$ by (3.12). Returning to (3.1) we then have (suppressing $t$ from notation)

$$
\dot{\omega}_{k}=\operatorname{Res}_{\zeta=\omega_{k}}\left[\left(\zeta P^{*}(\zeta)-\zeta R(\zeta)\right)(\log g(\zeta))^{\prime}\right],
$$

where we have to compute the right member. The residue of the first term is immediate:

$$
\operatorname{Res}_{\zeta=\omega_{k}} \zeta P^{*}(\zeta)(\log g(\zeta))^{\prime}=\omega_{k} P^{*}\left(\omega_{k}\right) .
$$

For the second term, there is a slight difficulty due to the fact that both factors in the product $R(\zeta)(\log g(\zeta))^{\prime}$ have poles at $\zeta=\omega_{k}$. However, we can use that

$$
(\log R)^{\prime}+(\log g)^{\prime}+\left(\log g^{*}\right)^{\prime}=0
$$

by the definition of $R$, hence that

$$
\begin{aligned}
\zeta R(\zeta)(\log g(\zeta))^{\prime} & =-\zeta R(\zeta)(\log R(\zeta))^{\prime}-\zeta R(\zeta)\left(\log g^{*}(\zeta)\right)^{\prime} \\
& =-\zeta R^{\prime}(\zeta)+\zeta^{-1} R(\zeta)(\log g(\zeta))^{\prime *} \\
& =-(\zeta R(\zeta))^{\prime}+R(\zeta)\left(1+\zeta^{-1}(\log g(\zeta))^{\prime *}\right) \\
& =-(\zeta R(\zeta))^{\prime}+R(\zeta)\left(1+\sum_{j}^{m} \frac{1}{1-\bar{\omega}_{j} \zeta}-\sum_{j}^{n} \frac{1}{1-\bar{\zeta}_{j} \zeta}\right)
\end{aligned}
$$

Here we used (2.18) in the last step. 
In the last expression, the first term has no residues since it is a pure derivative, and in the second term only $R(\zeta)$ has a pole (a simple one) at $\omega_{k}$. The residue of $R(\zeta)$ is

$$
\operatorname{Res}_{\zeta=\omega_{k}} R(\zeta)=\frac{2 q}{g^{\prime}\left(\omega_{k}\right) g^{*}\left(\omega_{k}\right)}=2 A_{k}
$$

hence we get

$$
\operatorname{Res}_{\zeta=\omega_{k}} \zeta R(\zeta)(\log g(\zeta))^{\prime}=2 A_{k}\left(1+\sum_{j=1}^{m} \frac{1}{1-\bar{\omega}_{j} \omega_{k}}-\sum_{j=1}^{n} \frac{1}{1-\bar{\zeta}_{j} \omega_{k}}\right) .
$$

Now (3.20) follows. The other equation, (3.21), is immediate.

It is easy to check that (3.21) is valid also in the case of multiple poles (and zeros). An immediate consequence is that, as has previously been observed in for example [20], [5], [7], poles always move away from the origin. More precisely we have

Corollary 3.1. For each $1 \leq j \leq n$ we have, when $q(t)>0$,

$$
\frac{d}{d t} \log \left|\zeta_{j}(t)\right|=\operatorname{Re} P^{*}\left(\zeta_{j}, t\right)>0
$$

along with the estimates

$$
\frac{\left|\zeta_{j}(t)\right|-1}{\left|\zeta_{j}(t)\right|+1} \leq \frac{d \log \left|\zeta_{j}(t)\right|}{d \log a_{1}(t)} \leq \frac{\left|\zeta_{j}(t)\right|+1}{\left|\zeta_{j}(t)\right|-1}
$$

Proof. It remains only to prove (3.23), and since

$$
\frac{d \log \left|\zeta_{j}(t)\right|}{d \log a_{1}(t)}=\frac{d \log \left|\zeta_{j}(t)\right| / d t}{d \log a_{1}(t) / d t}=\frac{\operatorname{Re} P^{*}\left(\zeta_{j}, t\right)}{P(0)}=\frac{\operatorname{Re} P\left(\zeta_{j}^{*}, t\right)}{P(0)}
$$

this follows easily from the ordinary Harnack inequalities for the positive harmonic function $\operatorname{Re} P(\zeta, t)$ in $\mathbf{D}$, applied at the point $\zeta=\zeta_{j}^{*}$.

Because of the middle term in the right member of (3.16), or the second term in (3.20), the zeros show up a more complicated behavior than the poles, and in particular they do not always move away from the origin (see next section). However, when $m>n$ they collectively move out, in fact even faster than the poles. This follows by taking the real part of (3.5) and using (3.13), (3.14), (3.18) and the definition (3.6) of $A_{\infty}$ :

\section{Proposition 3.2.}

$$
\frac{d}{d t} \sum_{k=1}^{m} \log \omega_{k}(t)-\frac{d}{d t} \sum_{j=1}^{n} \log \zeta_{j}(t)= \begin{cases}2\left(\int d \mu-A_{\infty}\right) & \text { if } m=n \\ (m-n+2) \int d \mu & \text { if } m>n\end{cases}
$$

In particular, if $m>n$,

$$
\begin{gathered}
\frac{d}{d t} \sum_{k=1}^{m} \log \left|\omega_{k}(t)\right|>\frac{d}{d t} \sum_{j=1}^{n} \log \left|\zeta_{j}(t)\right|, \\
\frac{d}{d t} \sum_{k=1}^{m} \arg \omega_{k}(t)=\frac{d}{d t} \sum_{j=1}^{n} \arg \zeta_{j}(t) .
\end{gathered}
$$


The case $m=n$ is indeed a little exceptional in that $f(\zeta, t)$ in this case has a simple pole at infinity which may be lost at one moment of time (see discussion after (2.15)), causing $m$ to temporarily drop below $n$. What happens in (2.15) then is that (at least) one root $\omega_{k}(t)$ rushes to infinity while $b(t)$ goes to zero. Then also $A_{\infty}$ goes to infinity. However, after the event the root comes back again.

Example 3.2. The following example of an off-center injection/suction of a disk is similar to examples which have been given by Hohlov, Howison, Richardson and others, see [8], [18]. We consider a Hele-Shaw evolution with $q(t)>0$ such that at one particular instant, which we choose to be $t=2$, the fluid domain is $\Omega(2)=\mathbf{D}(1,2)$. Then for a general $t$ the domain $\Omega(t)$ will satisfy the quadrature identity

$$
\int_{\Omega(t)} h d m=2 \pi(Q(t)-Q(2)) h(0)+4 \pi h(1)
$$

for functions $h$ harmonic and integrable in the domain. The mapping function at $t=2$ is

$$
f(\zeta, 2)=\frac{3 \zeta}{2-\zeta}
$$

while it for $t \neq 2$ can be taken to be of the form

$$
f(\zeta, t)=b(t) \frac{\zeta(\zeta-a(t))}{\zeta-t}
$$

for suitable real-valued functions $a(t)$ and $b(t)$. Here we have left the exact choice of $q(t)$ open in order to allow the time coordinate simply to be the location of the pole on the positive real axis, which by Corollary 3.1 is consistent with the assumption $q(t)>0$. The range of $t$ will then be $1<t<\infty$.

The derivative of $f$ is

$$
g(\zeta, t)=b(t) \frac{\zeta^{2}-2 t \zeta+t a(t)}{(\zeta-t)^{2}}
$$

which has two zeros $\omega_{1}(t), \omega_{2}(t)$ satisfying

$$
\left\{\begin{array}{l}
\omega_{1} \omega_{2}=t a(t), \\
\omega_{1}+\omega_{2}=2 t .
\end{array}\right.
$$

Using the fixed data, contained in the last term in (3.24), one gets the equations

$$
\begin{aligned}
f\left(t^{-1}, t\right) & =\frac{b(1-a t)}{t\left(1-t^{2}\right)}=1, \\
\operatorname{Res}_{\zeta=1 / t} f^{*}(\zeta, t) g(\zeta, t) d \zeta & =\frac{b^{2}\left(1-2 t^{2}+a t^{3}\right)(a-t)}{t\left(1-t^{2}\right)^{2}}=4,
\end{aligned}
$$

which can be solved for $a, b$, giving

$$
\begin{aligned}
& a(t)=\frac{1}{t}+\frac{\left(t^{2}-1\right)\left(t^{2}+1+\sqrt{\left(t^{2}-1\right)^{2}+16}\right)}{2 t\left(t^{2}-4\right)}, \\
& b(t)=\frac{t}{2}\left(t^{2}+1-\sqrt{\left.\left(t^{2}-1\right)^{2}+16\right)} .\right.
\end{aligned}
$$

Now the behavior of the roots can be read off from (3.25): for $1<t<2$ we have $\omega_{1} \omega_{2}=a t<0$, tending to $-\infty$ as $t \rightarrow 2$, while $\omega_{1}+\omega_{2}=2 t$ remains finite. Also, 
$b(t) \rightarrow 0$ as $t \rightarrow 2$. Thus the two roots go to infinity along the real axis (in different directions).

For $2<t<\infty, \omega_{1} \omega_{2}=a t>0$, which means that the roots come back along the imaginary axis as $t$ increases from 2 . Finally, as $t \rightarrow \infty$, they turn back to infinity the same way.

\section{Global locally univalent solutions are univalent}

In this section we show that any global locally univalent solution of the Polubarinova-Galin equation actually has to be univalent all the time. It is intuitively clear that it has to be so. In fact, if univalence breaks down while local univalence still holds, then this means that two different parts of $f(\mathbf{D}, t)$ start to overlap, hence a hole in the part of $\mathbf{C}$ covered by $f(\mathbf{D}, t)$ is created. With the solution being global this hole eventually has to be filled in, but it is easy to realize that when this occurs a branch point for the covering map from the multiply sheeted $f(\mathbf{D}, t)$ to $\mathbf{C}$ is created. In other words, a zero of $f^{\prime}$ moves into $\mathbf{D}$ and local univalence is lost.

A rigorous proof of the above statement can be based on a result from Löwner theory, namely Theorem 6.2 in [15]. To prepare for that, recall that in terms of the Taylor expansion $(2.10)$ of $f(\zeta, t)$ the zeroth order moment is given by

$$
M_{0}(t)=\sum_{j=1}^{\infty} j\left|a_{j}(t)\right|^{2} .
$$

Let

$$
N_{0}(t)=\sum_{j=2}^{\infty} j\left|a_{j}(t)\right|^{2}
$$

denote what remains after the first term. Then

$$
N_{0}(t)=M_{0}(t)-a_{1}(t)^{2}=M_{0}(0)+2 Q(t)-a_{1}(t)^{2}
$$

(recall $(2.5))$.

In [11] Kuznetsova showed that $N_{0}(t)$ is a decreasing function of $t$, hence that $a_{1}(t)^{2}-2 Q(t)$ is increasing and that

$$
\begin{aligned}
0 & \leq N_{0}(t) \leq N_{0}(0), \\
a_{1}(t)^{2} & \geq a_{1}(0)^{2}+2 Q(t) .
\end{aligned}
$$

It also follows that

$$
\frac{a_{1}(t)}{\sqrt{M_{0}(0)+2 Q(t)}}=\frac{a_{1}(t)}{\sqrt{M_{0}(t)}} \nearrow 1 \text { as } t \rightarrow \infty
$$

and that

$$
\left|a_{k}(t)\right| \leq \sqrt{\frac{N_{0}(0)}{k}} \text { for } k \geq 2 .
$$

Theorem 4.1. Let $f(\zeta, t) \in \mathcal{O}_{\text {locu }}(\overline{\mathbf{D}})$ be a global $(0 \leq t<\infty)$ locally univalent solution of the Polubarinova-Galin equation (2.1) driven by injection with rate $q(t)>$ 0 such that $Q(t) \rightarrow \infty$ as $t \rightarrow \infty$. Then $f(\zeta, t) \in \mathcal{O}_{\text {univ }}(\overline{\mathbf{D}})$ for all $0 \leq t<\infty$. 
Proof. The choice of time parameter is irrelevant as long as $Q(t) \rightarrow \infty$ as $t \rightarrow \infty$. Therefore, by (4.4), we may choose $t$ so that

$$
a_{1}(t)=e^{t} .
$$

Then $f(\zeta, t)$ solves the Löwner-Kufarev equation (2.6) with $P(\zeta, t)$ being a Carathéodory function, i.e., satisfying

$$
\operatorname{Re} P(\zeta, t)>0 \quad \text { in } \mathbf{D}, \quad P(0, t)=1 .
$$

This is the setting in [15].

Now, for (say) $|\zeta| \leq \frac{1}{2}$ and $t \geq 0$ we have, using (4.2),

$$
\begin{aligned}
|f(\zeta, t)| & \leq e^{t}+\sum_{j=2}^{\infty}\left|a_{j}(t)\right| 2^{-j} \leq e^{t}+\left(\sum_{j=2}^{\infty} j\left|a_{j}(t)\right|^{2}\right)^{1 / 2}\left(\sum_{j=2}^{\infty} \frac{1}{j} 2^{-2 j}\right)^{1 / 2} \\
& \leq e^{t}+\sqrt{N_{0}(t)} \leq e^{t}+\sqrt{N_{0}(0)}
\end{aligned}
$$

Thus $|f(\zeta, t)| \leq C e^{t}(0 \leq t<\infty)$ in a neighborhood of the origin, which means that the assumptions in Theorem 6.2 of [15] are satisfied. The conclusion is that $f(\zeta, t)$ is univalent for all $t \geq 0$. In our case it follows that $f(\zeta, t)$ is actually univalent in the closed unit disk, because by assumption it is locally univalent there, and if for two different points $\zeta_{1}, \zeta_{2} \in \partial \mathbf{D}$ we had $f\left(\zeta_{1}, t\right)=f\left(\zeta_{2}, t\right)$ for some $t$ then univalence in the open disk $\mathbf{D}$ would be lost in the next instance (the boundary is really propagating with positive speed under our assumptions).

Remark 4.1. Under the slightly weaker assumption that $f(\zeta, t) \in \mathcal{O}_{\text {norm }}(\overline{\mathbf{D}}) \cap$ $\mathcal{O}_{\text {locu }}(\mathbf{D})$ (i.e., local univalence only in the open disk) the theorem holds with the conclusion correspondingly changed to $f(\zeta, t) \in \mathcal{O}_{\text {univ }}(\mathbf{D})$.

\section{Asymptotic questions in the polynomial case}

In this section we study asymptotic behaviour of the roots of $g=f^{\prime}$ for locally univalent polynomial solutions of $(2.1)$ with $q(t)>0$ and $Q(t) \rightarrow \infty$ as $t \rightarrow \infty$. Thus $n=0$ and we write

$$
f(\zeta, t)=\sum_{j=1}^{m+1} a_{j}(t) \zeta^{j}
$$

We assume $a_{m+1}(0) \neq 0$, by which $a_{m+1}(t) \neq 0$ for all $t$, since $M_{m}=\overline{a_{m+1}(t)} a_{1}(t)^{m+1}$ (see (5.3) below) is preserved.

5.1. Collision of roots. In the rational case we have seen (Example 3.2) that the zeros of $g$ may occasionally (when $m=n$ ) move to infinity and come back again, and we have seen that the poles always move out. In the polynomial case $(n=0)$ the zeros cannot move to infinity in finite time, as can be seen from Proposition 3.2 for example. Below we give some examples showing that zeros can still move towards the origin and that they can collide, even though they in principle repel each other (as can be seen from (3.16) for example).

Example 5.1. When $m=2, n=0$ and the mapping function $f$ has real coefficients the solution of the Polubarinova-Galin equation can be made fully explicit (see Example 5.2). The roots $\omega_{1}$ and $\omega_{2}$ will either be a complex conjugate pair, or both will be real, with occasional collisions allowed. We shall first make some immediate 
conclusions from the dynamical equations (3.16) in the case that the roots $\omega_{1}, \omega_{2}$ are real.

Assume for example that both roots are positive, say

$$
1<\omega_{1}<\omega_{2} .
$$

By (3.8) the coefficients $A_{k}$ are then given by

$$
\begin{aligned}
\frac{|b|^{2}}{q} A_{1} & =\frac{\omega_{1}^{2}}{\left(\omega_{1}-\omega_{2}\right)\left(1-\omega_{1}^{2}\right)\left(1-\omega_{1} \omega_{2}\right)}, \\
\frac{|b|^{2}}{q} A_{2} & =\frac{\omega_{2}^{2}}{\left(\omega_{2}-\omega_{1}\right)\left(1-\omega_{2}^{2}\right)\left(1-\omega_{1} \omega_{2}\right)} .
\end{aligned}
$$

Now (3.16) together with (3.13) gives

where

$$
\begin{aligned}
& \frac{|b|^{2}}{q} \frac{d}{d t} \log \omega_{1}=\alpha\left(\omega_{1}, \omega_{2}\right)+\beta\left(\omega_{1}, \omega_{2}\right) \cdot \frac{\omega_{1} \omega_{2}-3}{\omega_{2}-\omega_{1}}, \\
& \frac{|b|^{2}}{q} \frac{d}{d t} \log \omega_{2}=\alpha\left(\omega_{1}, \omega_{2}\right)-\beta\left(\omega_{1}, \omega_{2}\right) \cdot \frac{\omega_{1} \omega_{2}-3}{\omega_{2}-\omega_{1}},
\end{aligned}
$$

$$
\begin{aligned}
& \alpha\left(\omega_{1}, \omega_{2}\right)=\frac{2\left(1+\omega_{1} \omega_{2}\right)}{\left(\omega_{1}^{2}-1\right)\left(\omega_{2}^{2}-1\right)\left(\omega_{1} \omega_{2}-1\right)}, \\
& \beta\left(\omega_{1}, \omega_{2}\right)=\frac{\left(\omega_{1}+\omega_{2}\right)}{\left(\omega_{1}^{2}-1\right)\left(\omega_{2}^{2}-1\right)\left(\omega_{1} \omega_{2}-1\right)} .
\end{aligned}
$$

Note that $\alpha\left(\omega_{1}, \omega_{2}\right)$ and $\beta\left(\omega_{1}, \omega_{2}\right)$ are positive and symmetric. The remaining factor $\frac{\omega_{1} \omega_{2}-3}{\omega_{2}-\omega_{1}}$ has a singularity when $\omega_{1}=\omega_{2}$ and changes sign when $\omega_{1} \omega_{2}=3$. Hence we can draw the following conclusions.

- The product $\omega_{1} \omega_{2}$ always increases in time (also clear from Proposition 3.2).

- The ratio $\omega_{2} / \omega_{1}$ decreases if $\omega_{1} \omega_{2}<3$, increases if $\omega_{1} \omega_{2}>3$

- If $\omega_{1} \omega_{2}<3$ and $\omega_{2}-\omega_{1}$ is sufficiently small, then $\dot{\omega}_{1}<0$ and $\dot{\omega}_{2}>0$. Hence no collisions occur for $\omega_{1} \omega_{2}<3$.

- If $\omega_{1} \omega_{2}=3$ then $\dot{\omega}_{1}=\dot{\omega}_{2}>0$.

- If $\omega_{1} \omega_{2}>3$ and $\omega_{2}-\omega_{1}$ is sufficiently small, then $\dot{\omega}_{1}>0$ and $\dot{\omega}_{2}<0$. The condition $\omega_{1} \omega_{2}>3$ is preserved in time and eventually leads to a collision (even if $\omega_{2}-\omega_{1}$ is not small to start with). After the collision the roots cease to be real and continue as a complex conjugate pair.

In the case of complex roots (but still with $f$ having real coefficients) the scenario may be quite interesting, as the following example due to Huntingford [10] shows.

Example 5.2. When expressed in terms of the moments $M_{1}, M_{2}$ and with the time parameter chosen so that $a_{1}(t)=e^{t}(q(t)>0$ defined accordingly), the solution of the Polubarinova-Galin equation (2.1) in the case $m=2, n=0$ with $f$ having real coefficients is explicitly

$$
f(\zeta, t)=e^{t} \zeta+\frac{M_{1} \zeta^{2}}{e^{2 t}+3 e^{-2 t} M_{2}}+e^{-3 t} M_{2} \zeta^{3}
$$

By taking

$$
\left\{\begin{array}{l}
M_{1}=\frac{32}{25}, \\
M_{2}=\frac{1}{5}
\end{array}\right.
$$


one gets an interesting example which has been investigated by Huntingford [10]. We let the solution start at the first possible instant of time, namely $t=t_{0}=\frac{1}{4} \log \frac{3}{5}<0$ (for smaller values of $t, f$ is not locally univalent). At that moment the image domain $\Omega(t)=f(\mathbf{D}, t)$ has two cusps on the boundary (the two zeros of $g$ form a complex conjugate pair on the unit circle). As $t$ increases these cusps resolve, the zeros of $g$ move out from the unit circle and collide on the real axis at some moment $t=t_{1}<0$. After that one of the zeros, say $\omega_{1}(t)$, moves back to the unit circle and reaches it again at time $t=0$. Thus a new cusp (which will be a $5 / 2$-power cusp) develops on the boundary. However also this cusp resolves, the root $\omega_{1}(t)$ moves away from the unit circle along the real axis, captures and collides with the other root $\omega_{2}(t)$, after which they leave the real axis and finally move towards infinity in the asymptotic directions of the positive and negative imaginary axes. All of this can be seen by examining [10] carefully. The final asymptotics follows from Theorem 5.1 below.

The solution is global $\left(t_{0}<t<\infty\right)$ and is all the time univalent (otherwise it would have broken down in finite time, by Theorem 4.1), or the remark following it.

The above example shows that it is possible for a single zero to reach the unit circle in the injection case. The next example will show that this can not occur if the other zeros are sufficiently separated and far away.

Example 5.3. Let $n=0, m \geq 2$ with $f$ allowed to have complex coefficients. We assume that one of the roots is close to the unit circle and the others far away and well separated from each other, say

$$
\begin{aligned}
1 & <\omega_{1}<1+\varepsilon, \quad \varepsilon>0 \text { small, } \\
\left|\omega_{k}-\omega_{j}\right| & \geq M \quad(1 \leq k, j \leq m, k \neq j, M>1 \text { large }) .
\end{aligned}
$$

We shall use equation (3.16) to investigate short time root behavior.

First observe by (3.8) that

$$
\frac{A_{k}}{\omega_{k}}=\frac{q}{|b|^{2}\left(1-\left|\omega_{k}\right|^{2}\right)} \cdot \frac{1}{\prod_{j \neq k}\left(\omega_{k}-\omega_{j}\right) \overline{\left(\omega_{k}^{*}-\omega_{j}\right)}},
$$

hence that, among these quantities, $\frac{A_{1}}{\omega_{1}}$ is the dominating one under the present assumptions. Therefore, by (3.16) and (3.13) (with $\left.A_{\infty}=0\right)$ we have

$$
\begin{aligned}
-\frac{d}{d t} \log \left|\omega_{1}(t)\right| & =\operatorname{Re} A_{0}+\operatorname{Re} \frac{2 A_{1}}{\omega_{1}}+\operatorname{Re} \sum_{j=2}^{m} \frac{2\left(A_{1}+A_{j}\right)}{\omega_{1}-\omega_{j}} \\
& \approx \operatorname{Re} \frac{3 A_{1}}{\omega_{1}}+\operatorname{Re}\left[\frac{2 A_{1}}{\omega_{1}} \sum_{j=2}^{m} \frac{\omega_{1}}{\omega_{1}-\omega_{j}}\right] \\
& \approx \operatorname{Re} \frac{3 A_{1}}{\omega_{1}}=\frac{3 q}{|b|^{2}\left(1-\left|\omega_{1}\right|^{2}\right)} \cdot \operatorname{Re} \frac{1}{\prod_{j=2}^{m}\left(\omega_{1}-\omega_{j}\right) \overline{\left(\omega_{1}^{*}-\omega_{j}\right)}} \\
& \approx \frac{3 q}{|b|^{2}\left(1-\left|\omega_{1}\right|^{2}\right) \prod_{j=2}^{m}\left|\omega_{1}-\omega_{j}\right|^{2}}<0,
\end{aligned}
$$

which shows that the minimum root $\omega_{1}(t)$ moves away from the origin to prevent the strong solution from blowing up in short time.

Similarly, in the case of suction, under the same assumptions the minimum root $\omega_{1}$ moves towards the origin initially and will make the solution blow up. 
5.2. Long time behaviour of roots and coefficients. We now assume that the locally univalent polynomial solution (5.1) is global in time. By Theorem 4.1, $f(\zeta, t)$ is then actually univalent all the time. We shall describe the asymptotic behavior of roots of $g(\zeta, t)$. It will be shown (Theorem 5.1) that these roots all move away from the origin as time $t$ is large, even though some of them may move towards the origin initially. One naturally expects that what makes the initial root behavior of $g(\zeta, t)$ unpredictable is that the distribution of zeros of $g(\zeta, 0)$ is not always uniform. Therefore, it seems reasonable that, by controlling the distribution of these initial roots, we can guarantee that all roots always move out. In fact, this will be demonstrated in Theorem 5.2.

Recall first Richardson's formula [17] for the harmonic moments $M_{k}$ (see (2.4)):

$$
M_{k}=\sum_{\left(i_{1}, \cdots, i_{k+1}\right)} i_{1} a_{i_{1}} \cdots a_{i_{k+1}} \overline{a_{i_{1}+\cdots+i_{k+1}}} .
$$

Here $k=0,1,2, \ldots$ and the summation runs over all $(k+1)$-tuples $\left(i_{1}, \cdots, i_{k+1}\right)$ of integers with $i_{j} \geq 1$, with the convention that $a_{j}=0$ for $j>m+1$ in the present polynomial case (5.1). See [17] and [11] for more details. Note that the final nonzero moment has a quite simple expression:

$$
M_{m}=a_{1}^{m+1} \overline{a_{m+1}} .
$$

Lemma 5.1 below will show that asymptotically, as $t \rightarrow \infty$, the formula (5.3) will almost be true for all moments.

Besides the zeros $\omega_{k}(t)$ of $g(\zeta, t)$, which are what we want to study, we introduce two other sets of zeros, for comparison:

- Let

$$
\tilde{\omega}_{k}(t)=\omega_{k}(t) a_{1}(t)^{-\frac{m+2}{m}}
$$

be corresponding rescaled zeros, namely the zeros of $\tilde{g}(\zeta, t)=g\left(a_{1}^{\frac{m+2}{m}} \zeta, t\right)$.

- Let

$$
\hat{\omega}_{k}=\frac{1}{\sqrt[m]{-(m+1) \bar{M}_{m}}}
$$

be the zeros of the polynomial $a_{1}+(m+1) a_{1}^{m+2} a_{m+1} \zeta^{m}$ consisting of the first and last term in $\tilde{g}(\zeta, t)$.

Note that the $\hat{\omega}_{k}$ do not depend on time. On the other hand, the rescaled zeros $\tilde{\omega}_{k}(t)$ are normalized in such a way that they asymptotically stabilize, and approach the $\hat{\omega}_{k}$ (Theorem 5.1). Note also that the product $\tilde{\omega}_{1}(t) \cdots \tilde{\omega}_{m}(t)=-\frac{1}{(m+1) \bar{M}_{m}}$ is a conserved quantity.

The following lemma is a slightly sharpened version of estimates first obtained in [12].

Lemma 5.1. Given $m \geq 1$ there exist universal polynomials $F_{j}\left(x_{1}, \ldots, x_{m+2}\right)$, $1 \leq j \leq m+1$, in $m+2$ real variables such that

$$
\left|a_{j}(t) a_{1}(t)^{j}-\overline{M_{j-1}}\right| \leq \frac{1}{a_{1}(t)^{4}} F_{j}\left(\frac{1}{a_{1}(0)}, \sqrt{N_{0}(0)},\left|M_{1}\right|, \cdots,\left|M_{m}\right|\right)
$$

$(t>0,1 \leq j \leq m+1)$ whenever $f(\zeta, t)$ given by (5.1) is a locally univalent solution of (2.1). The polynomials $F_{j}$ are increasing functions in all their variables. 
In particular we have estimates of the kind

$$
\begin{aligned}
\left|N_{0}(t)\right| & \leq \frac{C_{1}}{a_{1}(t)^{4}}, \\
\left|a_{j}(t)\right| & \leq \frac{C_{j}}{a_{1}(t)^{j}}, \quad 2 \leq j \leq m+1,
\end{aligned}
$$

for suitable constants $C_{j}=C_{j}\left(\frac{1}{a_{1}(0)}, \sqrt{N_{0}(0)},\left|M_{1}\right|,\left|M_{2}\right|, \cdots,\left|M_{m}\right|\right)$.

Proof. Using (5.2) we first prove by induction on decreasing $j$ that there exist polynomials $G_{j}\left(x_{1}, \ldots, x_{m+2}\right), 1 \leq j \leq m+1$, in $m+2$ variables, increasing in all variables, such that

$$
\left|a_{j}(t) a_{1}(t)^{j}-\overline{M_{j-1}}\right| \leq \frac{1}{a_{1}(t)^{2}} G_{j}\left(\frac{1}{a_{1}(0)}, \sqrt{N_{0}(0)},\left|M_{1}\right|, \cdots,\left|M_{m}\right|\right) .
$$

We start by setting $G_{m+1}=0$, which makes (5.8) hold for $j=m+1$. Now we show (5.8) for $j=k$ assuming it holds for all $j \geq k+1$. The arguments will work for any $1 \leq k \leq m$. Using (5.2) and suppressing dependence on $t$ from notation, we have

$$
\begin{aligned}
M_{k-1}-\bar{a}_{k} a_{1}^{k} & =\sum_{\left(i_{1}, \cdots, i_{k}\right) \neq(1, \ldots, 1)} i_{1} a_{i_{1}} \cdots a_{i_{k}} \cdot \overline{a_{i_{1}+\cdots+i_{k}}} \\
& =\sum_{\left(i_{1}, \cdots, i_{k}\right) \neq(1, \ldots, 1)} \frac{i_{1} a_{i_{1}} \cdots a_{i_{k}}}{a_{1}^{i_{1}+\cdots+i_{k}}} \cdot \overline{a_{i_{1}+\cdots+i_{k}}} a_{1}^{i_{1}+\cdots+i_{k}} \\
& =\frac{1}{a_{1}^{2}} \sum_{\left(i_{1}, \cdots, i_{k}\right) \neq(1, \ldots, 1)} \frac{i_{1} a_{i_{1}} \cdots a_{i_{k}}}{a_{1}^{i_{1}+\cdots+i_{k}-2}} \cdot \overline{a_{i_{1}+\cdots+i_{k}} a_{1}^{i_{1}+\cdots+i_{k}}} .
\end{aligned}
$$

We shall estimate the terms in the above sum. Since $\left(i_{1}, \cdots, i_{k}\right) \neq(1, \ldots, 1)$, at least one of the $i_{j}$ is $\geq 2$, hence it follows from (4.5), (4.3) that the first factors can be estimated as

$$
\left|\frac{i_{1} a_{i_{1}} \cdots a_{i_{k}}}{a_{1}^{i_{1}+\cdots+i_{k}-2}}\right| \leq(m+1) \sum_{0 \leq \alpha, \beta \leq m+1} \frac{\left(\sqrt{N_{0}(0)}\right)^{\alpha}}{a_{1}(0)^{\beta}} .
$$

Moreover, $i_{1}+\cdots+i_{k} \geq k+1$, so by the induction hypothesis we have

$$
\left|a_{i_{1}+\cdots+i_{k}} a_{1}^{i_{1}+\cdots+i_{k}}-\overline{M_{i_{1}+\cdots+i_{k}-1}}\right| \leq G_{i_{1}+\cdots+i_{k}}\left(\frac{1}{a_{1}(0)}, \sqrt{N_{0}(0)},\left|M_{1}\right|, \cdots,\left|M_{m}\right|\right) \frac{1}{a_{1}(t)^{2}},
$$

in particular,

$$
\left|a_{i_{1}+\cdots+i_{k}} a_{1}^{i_{1}+\cdots+i_{k}}\right| \leq G_{i_{1}+\cdots+i_{k}}\left(\frac{1}{a_{1}(0)}, \sqrt{N_{0}(0)},\left|M_{1}\right|, \cdots,\left|M_{m}\right|\right) \frac{1}{a_{1}(0)^{2}}+\sum_{j=1}^{m}\left|M_{j}\right| .
$$

Therefore we can estimate also the second factors in the above expression for $a_{k} a_{1}^{k}-$ $\overline{M_{k-1}}$. From this we easily deduce (5.8) for $j=k$ knowing that it is true for $j \geq k+1$. This completes the induction step and hence proves (5.8) for $1 \leq j \leq m$.

Now, already (5.8) shows that the estimate (5.7) holds. Since this estimate improves (4.5) by a factor at least $a_{1}(t)^{2}$ in the denominator, we can 'bootstrap' the previous argument: using the new estimate in (5.9) makes the induction process work with the factor $1 / a_{1}(t)^{2}$ in (5.8) replaced by $1 / a_{1}(t)^{4}$. Thus (5.5) follows, and since (5.6) is just the special case $j=1$ the lemma is proved. 
Theorem 5.1. With notations as above, assume that $f(\zeta, t)$ is a global polynomial solution of (2.1). Then

$$
\left|\tilde{\omega}_{k}(t)-\hat{\omega}_{k}\right| \rightarrow 0 \quad \text { as } t \rightarrow \infty
$$

if the roots $\tilde{\omega}_{k}(t)$ and $\hat{\omega}_{k}$ are ordered appropriately. Furthermore, all roots eventually move away from the origin as time is large enough.

Proof. The monic polynomial vanishing at the rescaled roots (5.4) is

$$
\begin{aligned}
\prod_{j=1}^{m}\left(\zeta-\tilde{\omega}_{j}\right) & =\sum_{j=1}^{m+1}\left(a_{1}^{-\frac{m+2}{m}}\right)^{m+1-j} \frac{j a_{j}}{(m+1) a_{m+1}} \zeta^{j-1} \\
& =\sum_{j=1}^{m+1} \frac{j}{m+1} \frac{a_{j} a_{1}^{j}}{a_{m+1} a_{1}^{m+1}} a_{1}^{-\frac{2}{m}(m+1-j)} \zeta^{j-1} \\
& =\frac{1}{(m+1) \overline{M_{m}}}+\sum_{j=2}^{m} \frac{j}{m+1} \frac{a_{j} a_{1}^{j}}{\overline{M_{m}}} a_{1}^{-\frac{2}{m}(m+1-j)} \zeta^{j-1}+\zeta^{m} .
\end{aligned}
$$

Due to Lemma 5.1 and because the exponents $-\frac{2}{m}(m+1-j)$ are strictly negative the coefficients of the middle terms tend to zero:

$$
\begin{aligned}
& \left|\frac{j}{m+1} \frac{a_{j} a_{1}^{j}}{M_{m}} a_{1}^{-\frac{2}{m}(m+1-j)}\right| \leq\left|\frac{j}{m+1} \frac{F_{j}}{a_{1}^{4} M_{m}} a_{1}^{-\frac{2}{m}(m+1-j)}\right| \\
& +\left|\frac{j}{m+1} \frac{M_{j-1}}{M_{m}} a_{1}^{-\frac{2}{m}(m+1-j)}\right| \rightarrow 0 \quad \text { as } t \rightarrow \infty \quad(2 \leq j \leq m) .
\end{aligned}
$$

From this it follows that $\left|\tilde{\omega}_{k}(t)-\hat{\omega}_{k}\right| \rightarrow 0$ as $t \rightarrow \infty$.

It also follows that the roots move away from the origin as time is large, because the speed of the roots only depends on the position of the roots, and it is clear that for a symmetric configuration of roots, like $\hat{\omega}_{k}$, the speed points radially away from the origin. A slightly more precise argument can be based on (3.20), (3.22), by which

$$
\frac{d}{d t} \log \left|\omega_{k}\right|=\operatorname{Re} P^{*}\left(\omega_{k}\right)-\operatorname{Re} \frac{2 q}{\omega_{k} g^{\prime}\left(\omega_{k}\right) g^{*}\left(\omega_{k}\right)}\left(1+\sum_{j=1}^{m} \frac{1}{1-\bar{\omega}_{j} \omega_{k}}\right) .
$$

Here the first term is always positive, while the subtracted term is negative whenever the roots are sufficiently far away and close to symmetrical configuration. In fact, in this case $\omega_{k} g^{\prime}\left(\omega_{k}\right) \approx-m a_{1}, g^{*}\left(\omega_{k}\right) \approx a_{1}$ and $1+\sum_{j=1}^{m} \frac{1}{1-\omega_{j} \omega_{k}} \approx 1$. (This argument will be made more precise in the proof of Theorem 5.2 below.) Hence $\frac{d}{d t} \log \left|\omega_{k}\right|>0$ for large $t$, as claimed.

5.3. Assumptions only on initial data. In this subsection we do not assume a priori that $f(\zeta, t)$ is global, we only make assumption on the initial data, and the solution being global will be part of the conclusion.

Theorem 5.2. In terms of any initial function $f(\zeta, 0)=\sum_{j=1}^{m+1} a_{j}(0) \zeta^{j}$, let $M>$ 0 be a common upper bound for $\left|M_{1}\right|, \ldots,\left|M_{m}\right|$ and $\sqrt{N_{0}(0)}$. Then, for any $\varepsilon>0$, there exists a number $B=B(\varepsilon, M, m)>0$ such that whenever $a_{1}(0)>B$ the polynomial solution $f(\zeta, t)$ starting with $f(\zeta, 0)$ is global in time and the assertions (i)-(iii) below hold for $1 \leq k \leq m$. 
(i) For a suitable ordering of the $\tilde{\omega}_{k}(t)$ and $\hat{\omega}_{k}$ we have

$$
\left|\tilde{\omega}_{k}(t)-\hat{\omega}_{k}\right|<\varepsilon, \quad 0 \leq t<\infty .
$$

In particular, the roots $\omega_{k}$ never collide (with $\varepsilon>0$ sufficiently small).

(ii)

$$
\left|\tilde{\omega}_{k}(t)-\hat{\omega}_{k}\right| \rightarrow 0 \quad \text { as } t \rightarrow \infty \text {. }
$$

(iii) The roots $\omega_{k}(t)$ move away from the origin (for all $t \geq 0$ ).

Proof. First, choose $\rho\left(M_{m}, m\right)>0$ so that the disks $\mathbf{D}\left(\hat{\omega}_{j}, \rho\right)(1 \leq j \leq m)$ are disjoint and such that, for any point $z \in \mathbf{D}\left(\hat{\omega}_{j}, \rho\right)$,

$$
\left|z^{m}+\frac{1}{(m+1) \overline{M_{m}}}\right|<\frac{1}{4(m+1)\left|M_{m}\right|} \tan \frac{\pi}{20} .
$$

For example, any $0<\rho<\frac{1}{4}\left|M_{m}\right|^{-\frac{1}{m}} \tan \frac{\pi}{20}$ will do.

Next, as in the proof of Theorem 5.1 we have that for any $0<\varepsilon<\rho$ there exists $\delta>0$ such that $\left|\tilde{\omega}_{k}-\hat{\omega}_{k}\right|<\varepsilon$ whenever

$$
\left|\frac{j}{m+1} \frac{a_{j} a_{1}^{j}}{\overline{M_{m}}} a_{1}^{-\frac{2}{m}(m+1-j)}\right|<\delta, \quad 2 \leq j \leq m .
$$

Finally, it follows from Lemma 5.1 that (5.12) indeed holds if just $a_{1}(0)$ is large enough. We conclude that for any $0<\varepsilon<\rho$, there exists $B_{1}(\varepsilon, M, m)>0$ such that if $a_{1}(0)>B_{1}$, then $\left|\tilde{\omega}_{k}(t)-\hat{\omega}_{k}\right|<\varepsilon$ and (5.11) holds. Therefore (i) holds. By Theorem 5.1, also (ii) holds.

From (3.20), (3.22) we see that in order to prove that all roots move away from the origin, it is sufficient to prove that

$$
\operatorname{Re}\left[\frac{2}{\omega_{k} g^{\prime}\left(\omega_{k}, t\right) g^{*}\left(\omega_{k}, t\right)}\left(1+\sum_{j=1}^{m} \frac{1}{1-\overline{\omega_{j}} \omega_{k}}\right)\right]<0 .
$$

We first estimate the terms of $\omega_{k} g^{\prime}\left(\omega_{k}, t\right)=\sum_{j=1}^{m} j(j+1) a_{j+1} \omega_{k}^{j}$. For $1 \leq j \leq m-1$ we have

$$
\begin{aligned}
a_{j+1} \omega_{k}^{j} & =a_{j+1}\left(\frac{\omega_{k}}{a_{1}^{\frac{m+2}{m}}}\right)^{j} a_{1}^{\frac{m+2}{m} j}=\left(\frac{\omega_{k}}{a_{1}^{\frac{m+2}{m}}}\right)^{j} \cdot a_{1}^{j+1} a_{j+1} \cdot a_{1}^{\frac{m+2}{m} j-(j+1)} \\
& =\tilde{\omega}_{k}^{j} \cdot a_{1}^{j+1} a_{j+1} \cdot a_{1}^{2\left(\frac{j}{m}-1\right)} a_{1} .
\end{aligned}
$$

Hence, for $1 \leq j \leq m-1$,

$$
\left|a_{j+1} \omega_{k}^{j}\right| \leq\left[1+\left(\varepsilon+\left|\hat{\omega}_{k}\right|\right)^{m-1}\right]\left(M+\frac{F_{j}}{a_{1}^{4}}\right) a_{1}^{-2 / m} a_{1}
$$

with $F_{j}=F_{j}\left(\frac{1}{a_{1}(0)}, M, \cdots, M\right)$ as in Lemma 5.1. From (5.14) and (4.3) we conclude that there exists $B_{2}>B_{1}$ such that if $a_{1}(0)>B_{2}$, then $\left|j(j+1) a_{j+1} \omega_{k}^{j}\right| \leq \frac{1}{4} a_{1} \tan \frac{\pi}{20}$. For $j=m, a_{j+1} \omega_{k}^{j}=a_{m+1} \omega_{k}^{m}=\tilde{\omega}_{k}^{m} \bar{M}_{j} a_{1}$ by (5.4).

When $a_{1}(0)>B_{2}$, we have

$$
\omega_{k} g^{\prime}\left(\omega_{k}, t\right)+m a_{1}=\left(\tilde{\omega}_{k}^{m}+\frac{1}{\bar{M}_{m}(m+1)}\right) \overline{M_{m}} m(m+1) a_{1}+\sum_{j=1}^{m-1} j(j+1) a_{j+1} \omega_{k}^{j}
$$


due to (5.11), and since $\left|j(j+1) a_{j+1} \omega_{k}^{j}\right| \leq \frac{1}{4} a_{1} \tan \frac{\pi}{20}$ we conclude from this that $\left|\arg \omega_{k}(t) g^{\prime}\left(\omega_{k}, t\right)-\pi\right|<\frac{\pi}{20}$. Finally, we can find $B=B(\delta, M, m)>B_{2}$ such that if $a_{1}(0)>B$, then $\left|\arg \omega_{k}(t) g^{\prime}\left(\omega_{k}, t\right)-\pi\right|,\left|\arg g^{*}\left(\omega_{k}, t\right)\right|$ and $\left|\arg \left(1+\sum_{j=1}^{m} \frac{1}{\omega_{j} \omega_{k}}\right)\right|$ are all $<\frac{\pi}{20}$. Therefore (5.13), and hence (iii) in the theorem, holds.

\section{Asymptotics for rational solutions}

In this section we study the asymptotics of poles and Taylor coefficients in the rational case. We assume that $f \in \mathcal{O}_{\text {locu }}(\overline{\mathbf{D}})$ and $g=f^{\prime}$ are of the forms $(2.20)$ and (2.15) and that $q(t)>0$ with $Q(t) \rightarrow \infty$ as $t \rightarrow \infty$. Recall (3.17), (3.21) and Corollary 3.1, which in particular show that the poles always move away from the origin. The following theorem gives more precise estimates of their locations.

Theorem 6.1. Assume $f(\zeta, t)$ is a global in time locally univalent solution of the Polubarinova-Galin equation, with $g(\zeta, t)$ rational of the form (2.15). Then $\left|\zeta_{j}(t)\right| \sim a_{1}(t)$ for each $1 \leq j \leq n$. More precisely, with $a_{1}(t)$ as in $(2.10)$

$$
\frac{1}{a_{1}(0)}\left(\left|\zeta_{j}(0)\right|+\frac{1}{\left|\zeta_{j}(0)\right|}-2\right) \leq \frac{\left|\zeta_{j}(t)\right|}{a_{1}(t)} \leq \frac{1}{a_{1}(0)}\left(\left|\zeta_{j}(0)\right|+\frac{1}{\left|\zeta_{j}(0)\right|}+2\right)
$$

for all $t \geq 0$.

Proof. Setting $\tau=\log a_{1}(t), \xi=\xi(\tau)=\left|\zeta_{j}(t)\right|>1$, the Harnack estimates (3.23) say that

$$
\frac{\xi(\xi-1)}{\xi+1} \leq \frac{d \xi}{d \tau} \leq \frac{\xi(\xi+1)}{\xi-1}
$$

On integrating the differential equations corresponding to the equality cases one easily obtains the inequalities

$$
\frac{1}{a_{1}(0)}\left(\left|\zeta_{j}(0)\right|+\frac{1}{\left|\zeta_{j}(0)\right|}-2\right) \leq \frac{1}{a_{1}(t)}\left(\left|\zeta_{j}(t)\right|+\frac{1}{\left|\zeta_{j}(t)\right|}-2\right)
$$

and

Since

$$
\frac{1}{a_{1}(t)}\left(\left|\zeta_{j}(t)\right|+\frac{1}{\left|\zeta_{j}(t)\right|}+2\right) \leq \frac{1}{a_{1}(0)}\left(\left|\zeta_{j}(0)\right|+\frac{1}{\left|\zeta_{j}(0)\right|}+2\right)
$$

$$
\frac{1}{a_{1}(t)}\left(\left|\zeta_{j}(t)\right|+\frac{1}{\left|\zeta_{j}(t)\right|}-2\right) \leq \frac{\left|\zeta_{j}(t)\right|}{a_{1}(t)} \leq \frac{1}{a_{1}(t)}\left(\left|\zeta_{j}(t)\right|+\frac{1}{\left|\zeta_{j}(t)\right|}+2\right)
$$

the desired estimates follow.

Next, turning to coefficients we shall write $f$ and $g$ as follows:

$$
\begin{aligned}
& f(\zeta, t)=\sum_{j=1}^{\ell} e_{j} \log \left(1-\frac{\zeta}{\zeta_{j}(t)}\right)+\frac{\sum_{j=1}^{m-\ell+1} b_{j}(t) \zeta^{j}}{\sum_{j=0}^{n-\ell} c_{j}(t) \zeta^{j}}=\sum_{j=1}^{\infty} a_{j}(t) \zeta^{j}, \\
& g(\zeta, t)=\frac{\sum_{j=0}^{m} \tilde{b}_{j}(t) \zeta^{j}}{\sum_{j=0}^{n} \tilde{c}_{j}(t) \zeta^{j}}=\sum_{j=0}^{\infty}(j+1) a_{j+1}(t) \zeta^{j} .
\end{aligned}
$$

Introduce also the Taylor coefficients $\tilde{a}_{j}$ of the pure rational part of $f(\zeta, t)$ by

$$
\frac{\sum_{j=1}^{m-\ell+1} b_{j}(t) \zeta^{j}}{\sum_{j=0}^{n-\ell} c_{j}(t) \zeta^{j}}=\sum_{j=1}^{\infty} \tilde{a}_{j}(t) \zeta^{j} .
$$


The above expansions are to be compared with (2.15) and (2.20). In particular, $\ell$ denotes the number of different (finite) poles of $g, n_{j}$ denotes the order of the pole at $\zeta_{j}($ as in $(2.15))$ and

$$
n=\ell+\sum_{j=1}^{\ell}\left(n_{j}-1\right)=\sum_{j=1}^{\ell} n_{j}
$$

is the total order of the finite poles of $g$. The numbers $e_{j}$ are the residues of $g(\zeta) d \zeta$ at the points $\zeta_{j}$, and some or all of them may be zero.

At infinity, $g(\zeta) d \zeta$ has a pole of order $n_{0}=m-n+2$ (as a differential), hence $f$ has a pole of order $m-n+1$ there. This is also what (6.1) gives, hence the notations in (6.1) and (6.2) are consistent with those in (2.20) and (2.15). Recall also that the coefficients $e_{j}$ do not depend on $t$. The coefficients $b_{j}, c_{j}, \tilde{a}_{j}, \tilde{b}_{j}, \tilde{c}_{j}$ satisfy $b_{m-\ell+1} \neq 0, c_{n-\ell} \neq 0, b_{1} \neq 0, c_{0} \neq 0, \tilde{b}_{m} \neq 0, \tilde{c}_{n} \neq 0, \tilde{a_{1}} \neq 0, \tilde{b}_{1} \neq 0, \tilde{c}_{0} \neq 0$, and we shall normalize them so that $c_{0}=1, \tilde{c}_{0}=1$.

Lemma 6.1. Assume that $f(\zeta, t)$, given by (6.1), is a global solution. Then, as $t \rightarrow \infty$,

$$
\begin{aligned}
\left|c_{n-\ell}\right| \sim a_{1}^{-(n-\ell)}, & \left|c_{j}\right| & =O\left(a_{1}^{-j}\right) \quad \text { for } 1 \leq j \leq n-\ell-1, \\
\left|b_{1}\right| \sim \tilde{a}_{1} \sim a_{1}, & \left|b_{j}\right| & =O(1) \quad \text { for } 2 \leq j \leq m-\ell+1, \\
\left|\tilde{c}_{n}\right| \sim a_{1}^{-n}, & \left|\tilde{c}_{j}\right| & =O\left(a_{1}^{-j}\right) \quad \text { for } 1 \leq j \leq n-1, \\
\left|\tilde{b}_{0}\right| \sim a_{1}, & \left|\tilde{b}_{j}\right| & =O(1) \quad \text { for } 1 \leq j \leq m .
\end{aligned}
$$

See Subsection 2.1 for the meaning of $\sim$.

Proof. Since $\sum_{j=0}^{n-\ell} c_{j}(t) \zeta^{j}=c_{n-\ell}(t) \prod_{j=1}^{n-\ell}\left(\zeta-\zeta_{j}(t)\right)$ and $c_{0}=1$ the estimates for $c_{j}(t)$ follow immediately from Theorem 6.1. Similarly for $\tilde{c}_{j}(t)$.

Since by (6.1), (6.3),

$$
a_{j}(t)=\tilde{a}_{j}(t)-\sum_{k=1}^{\ell} \frac{e_{k}}{j \zeta_{k}(t)^{j}}
$$

we have

$$
\left|\tilde{a}_{j}(t)-a_{j}(t)\right| \leq \frac{C_{j}}{a_{1}(t)^{j}} \rightarrow 0,
$$

as $t \rightarrow \infty$. The coefficients $b_{k}$ are given by

$$
b_{k}=\tilde{a}_{1} c_{k-1}+\sum_{j=2}^{k-1} \tilde{a}_{j} c_{k-j}+\tilde{a}_{k}
$$

and since the $a_{j}$, and hence the $\tilde{a}_{j}, j \geq 2$, are bounded (see (4.5) for example) the assertions about the $b_{j}$ follow easily. For the $\tilde{b}_{k}$ we have similarly

$$
\tilde{b}_{k}=a_{1} \tilde{c}_{k}+\sum_{j=1}^{k-1}(j+1) a_{j+1} \tilde{c}_{k-j}+(k+1) a_{k+1},
$$

hence the estimates for these follow in the same way. 
Theorem 6.2. Assume that $f(\zeta, t)$ in (6.1) is a global solution and introduce the truncations

$$
f_{N}(\zeta, t)=\sum_{j=1}^{N} a_{j}(t) \zeta^{j} .
$$

Then the following assertions hold.

(i) There exist numbers $s_{N}$ with $s_{N} \rightarrow \infty$ as $N \rightarrow \infty$ such that, for $j=0,1$,

$$
\sup _{\zeta \in \overline{\mathbf{D}}}\left|f_{N}^{(j)}(\zeta, t)-f^{(j)}(\zeta, t)\right|=O\left(a_{1}(t)^{-s_{N}}\right),
$$

as $t \rightarrow \infty$. The same is true for any $j \geq 0$, with $s_{N}$ then depending on $j$.

(ii) For each $k \geq 2$,

$$
\lim _{t \rightarrow \infty} a_{k}(t) a_{1}(t)^{k}=\bar{M}_{k-1} .
$$

(iii) Assume that, for some number $r \geq 2, M_{1}=\cdots=M_{r-1}=0, M_{r} \neq 0$. Then

$$
\lim _{t \rightarrow \infty} a_{s}(t) a_{1}(t)^{r+1}=0, \quad 2 \leq s \leq r,
$$

and $r \leq m$. In case there are no logarithmic singularities (i.e., $e_{j}=0$, $1 \leq j \leq \ell$ ) then we even have $r \leq m-\ell$.

Proof. Write $f$ on the form

$$
\begin{aligned}
f(\zeta, t) & =\sum_{j=1}^{\ell} e_{j} \log \left(1-\frac{\zeta}{\zeta_{j}}\right)+b_{1} \zeta \frac{1+P(\zeta)}{1-Q(\zeta)} \\
& =-\sum_{k=1}^{\infty}\left(\sum_{j=1}^{\ell} \frac{e_{j}}{k \zeta_{j}^{k}}\right) \zeta^{k}+b_{1} \zeta \sum_{k=0}^{\infty}\left(Q(\zeta)^{k}+P(\zeta) Q(\zeta)^{k}\right),
\end{aligned}
$$

namely with

$$
P(\zeta)=\sum_{j=1}^{m} \frac{b_{j+1}}{b_{1}} \zeta^{j}, \quad Q(\zeta)=-\sum_{j=1}^{n} c_{j} \zeta^{j} .
$$

Then all coefficients in $P$ and $Q$ are $O\left(a_{1}^{-1}\right)$ by Lemma 6.1 , hence

$$
\sup _{\mathbf{D}(0, R)}|P| \leq \frac{C_{R}}{a_{1}}, \quad \sup _{\mathbf{D}(0, R)}|Q| \leq \frac{C_{R}}{a_{1}}
$$

for any fixed $R>1$ and suitable constants $C_{R}$. It follows from the above that for large $N$ the remainder

$$
f(\zeta)-f_{N}(\zeta)=\sum_{j=N+1}^{\infty} a_{j} \zeta^{j}
$$

is built up by terms of the kind $\zeta Q(\zeta)^{k}$ and $\zeta P(\zeta) Q(\zeta)^{k}$ with also $k$ large. Considering only $t$ so large that $\frac{C_{R}}{a_{1}(t)} \leq \frac{1}{2}$ (for example) it follows that

$$
\sup _{\mathbf{D}(0, R)}\left|f-f_{N}\right| \leq \frac{C_{R}}{a_{1}^{s}}
$$

where $s$ can be made arbitrarily large by choosing $N$ sufficiently large. Now (6.6) follows. 
(ii) Recall the expression (2.4) for the moments $M_{k}=M_{k}(f)$ in terms of $f$. The truncations $f_{N}$ of $f$ similarly define moments:

$$
M_{k}\left(f_{N}\right)=\frac{1}{2 \pi i} \int_{\partial \mathbf{D}} f_{N}^{k}(\zeta, t) f_{N}^{\prime}(\zeta, t) f_{N}^{*}(\zeta, t) d \zeta=\sum_{\sum i_{j} \leq N} i_{1} a_{i_{1}} \cdots a_{i_{k+1}} \overline{a_{i_{1}+\cdots+i_{k+1}}} .
$$

It is clear from (2.4) that each moment $M_{k}(f)$ is a Lipschitz continuous function of $f$ if $f$ is measured by the norm $\sup _{\overline{\mathbf{D}}}\left(|f|+\left|f^{\prime}\right|\right)$, or any norm $\sup _{\mathbf{D}(0, R)}|f|, R>1$. Therefore we have, by choosing $N=N_{k}$ large enough in (6.6), with $j=0,1$ and on using (6.9), that for any exponent $p \geq 0$ we have

$$
\left|M_{k}(f)-M_{k}\left(f_{N}\right)\right| \leq C^{\prime}\left|f-f_{N}\right| \leq \frac{C^{\prime \prime}}{a_{1}^{p}}
$$

for some constants $C^{\prime}$ and $C^{\prime \prime}$ (which depend on $f$ and $p$ ). From this we get (with $\left.M_{k}=M_{k}(f)\right)$

$$
\begin{aligned}
a_{1}^{k+1} \overline{a_{k+1}}-M_{k} & =M_{k}\left(f_{N}\right)-M_{k}-\sum_{k+2 \leq \sum i_{j} \leq N} i_{1} a_{i_{1}} \cdots a_{i_{k+1}} \overline{a_{i_{1}+\cdots+i_{k+1}}} \\
& =O\left(\frac{1}{a_{1}^{p}}\right)-\sum_{k+2 \leq \sum i_{j} \leq N} i_{1} a_{i_{1}} \cdots a_{i_{k+1}} \overline{a_{i_{1}+\cdots+i_{k+1}}} \\
& =O\left(\frac{1}{a_{1}^{p}}\right)-(k+2) a_{1}^{k} a_{2} \overline{a_{k+2}}-\sum_{k+3 \leq \sum i_{j} \leq N} i_{1} a_{i_{1}} \cdots a_{i_{k+1}} \overline{a_{i_{1}+\cdots+i_{k+1}}} .
\end{aligned}
$$

Now, to prove (6.7) we shall prove by induction that for every $s \geq 2$, the two assertions

$$
\begin{aligned}
\lim _{t \rightarrow \infty} a_{1}(t)^{s} \overline{a_{s}(t)} & =M_{s-1}, \\
\left|a_{j}(t)\right| & =O\left(\frac{1}{a_{1}(t)^{s}}\right), \quad j \geq s,
\end{aligned}
$$

hold. First, on using the fact that the $\left|a_{j}(t)\right|, j \geq 2$, are uniformly bounded we deduce from (6.10) that

$$
\left|a_{1}^{k+1} \overline{a_{k+1}}\right| \leq\left|M_{k}\right|+O\left(\frac{1}{a_{1}^{p}}\right)+O\left(a_{1}^{k}\right), \quad k \geq 1,
$$

and hence that $\left|a_{k+1}\right|=O\left(\frac{1}{a_{1}}\right), k \geq 1$. Now repeating (6.10) with the new estimate $\left|a_{j}\right|=O\left(\frac{1}{a_{1}}\right), j \geq 2$, we obtain

$$
\left|a_{1}^{k+1} \overline{a_{k+1}}\right| \leq\left|M_{k}\right|+O\left(\frac{1}{a_{1}^{p}}\right)+O\left(a_{1}^{k-2}\right), \quad k \geq 1
$$

and hence $\left|a_{k+1}\right|=O\left(\frac{1}{a_{1}^{2}}\right), k \geq 1$.

To start the induction process, take $k=1$ and use the last estimate $\left|a_{j}\right|=O\left(\frac{1}{a_{1}^{2}}\right)$, $j \geq 2$, in (6.10). This gives

$$
a_{1}^{2} \overline{a_{2}}-M_{1}=O\left(\frac{1}{a_{1}^{p}}\right)+O\left(\frac{1}{a_{1}^{3}}\right)
$$

and hence $\lim _{t \rightarrow \infty} a_{1}^{2} \overline{a_{2}}=M_{1}$. Therefore (6.11) and (6.12) hold for $s=2$. 
Now take $s_{0} \geq 2$ and assume that (6.11), (6.12) hold for all $s \leq s_{0}$. Then we shall prove (6.11), (6.12) for $s=s_{0}+1$. Thus we may in (6.10) use the fact that for any $s \leq s_{0}$ we have $\left|a_{j}\right|=O\left(\frac{1}{a_{1}^{s}}\right), j \geq s$. This gives

$$
\left|a_{1}^{k+1} a_{k+1}\right| \leq\left|M_{k}\right|+O\left(\frac{1}{a_{1}^{p}}\right)+O\left(a_{1}^{k-2-s_{0}}\right), \quad k \geq s_{0},
$$

and hence $\left|a_{k+1}\right|=O\left(\frac{1}{a_{1}^{s_{0}+1}}\right)$ for $k+1 \geq s_{0}+1$. It follows that (6.12) holds for $s=s_{0}+1$.

Using, in (6.10) with $k=s_{0}$, that $\left|a_{j}\right|=O\left(\frac{1}{a_{1}^{s_{0}+1}}\right)$ for $j \geq s_{0}+1$ (just proved) and $\left|a_{j}\right|=O\left(\frac{1}{a_{1}^{j}}\right)$ for $2 \leq j \leq s_{0}$ (induction hypothesis), we obtain

$$
a_{1}^{s_{0}+1} \overline{a_{s_{0}+1}}=M_{s_{0}}+O\left(\frac{1}{a_{1}^{p}}\right)+O\left(\frac{1}{a_{1}^{3}}\right)
$$

and hence $\lim _{t \rightarrow \infty} a_{1}^{s_{0}+1} \overline{a_{s_{0}+1}}=M_{s_{0}}$. Therefore also (6.11) holds for $s=s_{0}+1$. Thus (ii) in the theorem is proved.

(iii) Assuming now $M_{1}=\cdots=M_{r-1}=0$, (6.10) with $1 \leq k \leq r-1$ gives

$$
\begin{aligned}
\overline{a_{k+1}} & =O\left(\frac{1}{a_{1}^{p+k+1}}\right)-\sum_{k+2 \leq \sum i_{j} \leq N} i_{1} \frac{a_{i_{1}} \cdots a_{i_{k+1}}}{a_{1}^{k+1}} \overline{a_{i_{1}+\cdots+i_{k+1}}} \\
& =O\left(\frac{1}{a_{1}^{p+k+1}}\right)-(k+2) \frac{a_{2}}{a_{1}} \cdot \overline{a_{k+2}}-\sum_{k+3 \leq \sum i_{j} \leq N} i_{1} \frac{a_{i_{1}} \cdots a_{i_{k+1}}}{a_{1}^{k+1}} \overline{a_{i_{1}+\cdots+i_{k+1}}}
\end{aligned}
$$

with $p$ arbitrarily large. Using (6.7) and choosing $p \geq 4$, the above gives

$$
a_{k+1}=O\left(\frac{1}{a_{1}^{p+k+1}}\right)+O\left(\frac{1}{a_{1}^{k+5}}\right)=O\left(\frac{1}{a_{1}^{k+5}}\right) .
$$

With $k=r-1$ this gives (6.8) for $s=r$. It also follows for $s=r-2$. Assume now, as an induction hypothesis, that (6.8) holds for $s_{0} \leq s \leq r$. If $s_{0} \geq 3$, we then let $k=s_{0}-2$ in (6.13), and use (6.7) and the induction hypothesis. This gives (6.8) for $s=s_{0}-1$. Hence (6.8) is proved.

The identity (6.5) holds for $1 \leq k \leq m$, but also for $k>m$ with the convention that $b_{k}=0$ when $k>m$, and $\tilde{c}_{j}=0$ when $j>n$. Choosing $k=m+1$ then gives

$$
(m+2) a_{m+2}=-\sum_{j=1}^{m}(j+1) a_{j+1} \tilde{c}_{m+1-j} .
$$

If $r \geq m+1$ then (6.8) and Lemma 6.1 show that the right member is $o\left(a_{1}^{-(r+1)}\right.$. $\left.O\left(a_{1}^{-1}\right)\right)=o\left(a_{1}^{-(r+2)}\right)$, while (6.7) shows that the left member is exactly $O\left(a_{1}^{-(r+2)}\right)$, if $M_{r} \neq 0$. This contradiction shows that $r \leq m$.

Similarly, using (6.4) one obtains $r \leq m-\ell$ in case there are no logarithmic terms in $f$. An alternative way of proving these upper bounds for $r$ is given in the remark below.

Remark 6.1. The fact that for rational $g$, the vanishing of a sufficiently long sequence $M_{1}, M_{2}, \ldots, M_{k}$ of moments implies the vanishing of all $M_{j}, j \geq 1$, can also be deduced from the relevant quadrature identity, like (2.14), holding for the image domain. Assume for example that $g(\zeta) d \zeta$ has no residues, so that there are no line 
integrals in (2.14). Then choosing $h(\zeta)=\frac{1}{z-\zeta}$ with $z \in \mathbf{C} \backslash \bar{\Omega}$ in (2.14) gives the identity

$$
\sum_{k=0}^{\infty} \frac{M_{k}}{z^{k+1}}=\sum_{j=0}^{\ell} \sum_{k=1}^{n_{j}-1} \frac{k ! a_{j k}}{\left(z-z_{j}\right)^{k}}=\frac{\sum_{j=0}^{m-\ell} B_{j} z^{j}}{\sum_{j=0}^{m-\ell+1} C_{j} z^{j}}
$$

for suitable $B_{j}, C_{j}$. Recall that $\sum_{j=0}^{\ell}\left(n_{j}-1\right)=m-\ell+1$, see at $(2.14)$ and $(2.20)$. If now $M_{1}=\ldots M_{r-1}=0, M_{r} \neq 0$, then we get

$$
\left(M_{0}+\frac{M_{r}}{z^{r}}+\frac{M_{r+1}}{z^{r+1}}+\ldots\right)\left(C_{m-\ell+1}+\frac{C_{m-\ell}}{z}+\cdots+\frac{C_{0}}{z^{m-\ell+1}}\right)=B_{m-\ell}+\frac{B_{m-\ell-1}}{z}+\cdots+\frac{B_{0}}{z^{m-\ell}} .
$$

Here it is easy to see from the general structure (2.15), (2.20) that $M_{0} \neq 0, C_{m-\ell+1} \neq$ $0, B_{m-\ell} \neq 0$. On the other hand, $C_{0}=C_{1}=\cdots=C_{n_{0}-1}=0$ because the rational function above has a pole of order (exactly) $n_{0}-1$ at $z_{0}=0$. Thus, in view of the fact that $m-\ell+1-\left(n_{0}-1\right)=n-\ell$ the left hand side of the above equation actually is

$$
\begin{aligned}
& \left(M_{0}+\frac{M_{r}}{z^{r}}+\frac{M_{r+1}}{z^{r+1}}+\ldots\right)\left(C_{m-\ell+1}+\frac{C_{m-\ell}}{z}+\cdots+\frac{C_{n_{0}}}{z^{m-\ell+1-\left(n_{0}-1\right)}}\right) \\
& =M_{0} C_{m-\ell+1}+\frac{M_{0} C_{m-\ell}}{z}+\cdots+\frac{M_{0} C_{n_{0}}}{z^{n-\ell}}+\frac{C_{m-\ell+1}}{z^{r}}+O\left(\frac{1}{z^{r+1}}\right)+\ldots
\end{aligned}
$$

Comparing with the right hand and using that $n-\ell \leq m-\ell$ it follows that $r$ cannot be larger than $m-\ell$, which is also what Theorem 6.2 tells. $f$.

In a similar way one proves that $r \leq m$ in the presence of logarithmic terms in

From Theorem 6.2, we obtain the following result:

Corollary 6.2. Let $f(\zeta, t)$ be a global solution of the form (6.1) and assume $M_{1}=\cdots=M_{r-1}=0, M_{r} \neq 0$. Then,

$$
\lim _{t \rightarrow \infty} \sup _{\zeta \in \partial \mathbf{D}}\left|\left[f(\zeta, t)-\sqrt{2 Q(t)+M_{0}(0)} \zeta\right](\sqrt{2 Q(t)})^{r+1}-\overline{M_{r}} \zeta^{r+1}\right|=0 .
$$

Proof. By (4.1), (4.2), (4.3) and Theorem 6.2,

$$
a_{1}(t)-\sqrt{2 Q(t)+M_{0}(0)}=\frac{-N_{0}(t)}{a_{1}(t)+\sqrt{2 Q(t)+M_{0}(0)}}=O\left(\frac{1}{a_{1}^{2 r+3}}\right) .
$$

Applying once more (4.2), (4.3) and Theorem 6.2 the corollary follows.

\section{References}

[1] Abanov, A., M. Mineev-Weinstein, and A. Zabrodin: Multi-cut solutions of Laplacian growth. - Phys. D 238:17, 2009, 1787-1796.

[2] Dawson, S. P., and M. Mineev-Weinstein: Long-time behavior of the $N$-finger solution of the Laplacian growth equation. - Phys. D 73:4, 1994, 373-387.

[3] Gustafsson, B.: On a differential equation arising in a Hele-Shaw flow moving boundary problem. - Ark. Mat. 22:2, 1984, 251-268.

[4] Gustafsson, B., and Y.-L. Lin: Non-univalent solutions Polubarinova-Galin equation. - In preparation.

[5] Gustafsson, B., and D. Prokhorov, and A. Vasil'ev: Infinite lifetime for the starlike dynamics in Hele-Shaw cells. - Proc. Amer. Math. Soc. 132:9, 2004, 2661-2669 (electronic). 
[6] Gustafsson, B., and H.S. Shapiro: What is a quadrature domain? - In: Quadrature domains and their applications, Oper. Theory Adv. Appl. 156, Birkhäuser, Basel, 2005, 1-25.

[7] Gustafsson, B., and A. Vasil'ev: Conformal and potential analysis in Hele-Shaw cells. Adv. Math. Fluid Mech., Birkhäuser Verlag, Basel, 2006.

[8] Hohlov, Y.E., and S. D. Howison: On the classification of solutions to the zero-surfacetension model for Hele-Shaw free boundary flows. - Quart. Appl. Math. 51:4, 1993, 777-789.

[9] Howison, S. D.: Cusp development in Hele-Shaw flow with a free surface. - SIAM J. Appl. Math. 46:1, 1986, 20-26.

[10] Huntingford, C.: An exact solution to the one-phase zero-surface-tension Hele-Shaw freeboundary problem. - Comput. Math. Appl. 29:10, 1995, 45-50.

[11] Kuznetsova, O. S.: On polynomial solutions of the Hele-Shaw problem. - Sibirsk. Mat. Zh. 42:5, 2001, 1084-1093.

[12] LIN, Y.-L.: Large-time rescaling behaviours of Stokes and Hele-Shaw flows driven by injection. - European J. Appl. Math. 22:1, 2011, 7-19.

[13] Lin, Y.-L.: Perturbation theorems for Hele-Shaw flows and their applications. - Ark. Mat. 49, 2011, 357-382.

[14] Mineev, M. B.: A finite polynomial solution of the two-dimensional interface dynamics. Phys. D 43:2-3, 1990, 288-292.

[15] Pommerenke, Ch.: Univalent functions. - Studia Mathematica/Mathematische Lehrbücher, Band XXV, Vandenhoeck \& Ruprecht, Göttingen, 1975.

[16] Reissig, M., and von Wolfersdorf, L.: A simplified proof for a moving boundary problem for Hele-Shaw flows in the plane. - Ark. Mat. 31:1, 1993, 101-116.

[17] Richardson, S.: Hele-Shaw flows with a free boundary produced by the injection of fluid into a narrow channel. - J. Fluid Mech. 56, 1972, 609-618.

[18] Richardson, S.: On the classification of solutions to the zero-surface-tension model for HeleShaw free boundary flows. - Quart. Appl. Math. 55:2, 1997, 313-319.

[19] Shraiman, B., and D. Bensimon: Singularities in nonlocal interface dynamics. - Phys. Rev. A (3) 30:5, 1984, 2840-2842.

[20] Tanveer, S.: Evolution of Hele-Shaw interface for small surface tension. - Philos. Trans. R. Soc. Lond. Ser. A Math. Phys. Eng. Sci. 343:1668, 1993, 155-204.

[21] Varchenko, A. N., and P. I. Etingof: Why the boundary of a round drop becomes a curve of order four. - Univ. Lecture Ser., Amer. Math. Soc., Providence, Rhode Island, third ed., 1992 .

[22] Vondenhoff, E.: Long-time asymptotics of Hele-Shaw flow for perturbed balls with injection and suction. - Interfaces Free Bound. 10:4, 2008, 483-502.

Received 26 March $2012 \bullet$ Accepted 6 June 2012 